\title{
Gemeinsame Risikofaktoren von depressiver und ängstlicher Symptomatik im Kindes- und Jugendalter: ein systemati- sches Review aus transdiagnostischer Perspektive
}

\section{Common Risk Factors of Depressive and Anxiety Symptomatics in Childhood and Adolescence: A Systematic Review from Transdiagnostic Perspectives}

Autoren

Institut
E. Belhadj Kouider, F. Petermann

Zentrum für Klinische Psychologie und Rehabilitation, Universität Bremen
Schlüsselwörter

- Depression

- Angststörungen

- Risikofaktoren

- Kinder und Jugendliche

- Transdiagnostik

Key words

- depression

- anxiety disorders

- risk factors

- children and adolescents

- transdiagnostics
Bibliografie

DOI http://dx.doi.org/

10.1055/s-0035-1553089

Fortschr Neurol Psychiatr 2015;

83: 321-333 @ Georg Thieme

Verlag KG Stuttgart . New York .

ISSN 0720-4299

Korrespondenzadresse

Esmahan Belhadj Kouider

Zentrum für Klinische

Psychologie und Rehabilitation, Universität Bremen

Grazer Str. 6

28359 Bremen

e.belhadj@uni-bremen.de

\section{Zusammenfassung}

$\nabla$

Die transdiagnostische Perspektive verweist auf gemeinsame Ursachen von psychischen Störungen. Die vorliegende Studie recherchiert in diesem Kontext weiterführend zur Studie von Epkins und Heckler systematisch gemeinsame Risikofaktoren für depressive und ängstliche Symptomatik bei Kindern und Jugendlichen. Es konnten zwischen 2012 und 2014 insgesamt 35 Studien identifiziert werden, die 44 gemeinsame Risikofaktoren für diese internalisierende Symptomatik im Kindesund Jugendalter aufzeigen können. Das weibliche Geschlecht, das Einsetzen der Pubertät, der Umgang mit chronischen Erkrankungen, erhöhtes Stresserleben von Kindern oder Müttern, negatives Erziehungsverhalten der Eltern, Missbrauch und abweisende Erfahrungen von Gleichaltrigen sind Faktoren, die in der Entwicklungspsychopathologie von Depression und Angststörungen im Kindes- und Jugendalter relevant sind. Die gemeinsamen Risikofaktoren legen eine ähnliche Behandlung dieser Krankheiten nahe.

\section{Einleitung}

$\nabla$

Angststörungen und Depressionen gehören zu den häufigsten psychischen Erkrankungen im Kindes- und Jugendalter und können als Risikofaktoren für psychische Störungen im Erwachsenenalter gelten [1-3]. Diese beiden Störungen sind gekennzeichnet durch eine Symptomatik, bei der Kinder oder Jugendliche dazu neigen, ihre Probleme oder ihren Stress nicht nach außen zu tragen und mitzuteilen, sondern vermehrt nach innen zu richten $[4,5]$. Emotionale Schwierigkeiten und Vermeidungsverhalten stehen bei der internalisierenden Symptomatik im Vordergrund $[4,6]$. Das DSM-5 [7] beschreibt beispielsweise bei Angststörungen die intensive und extrem ausgeprägte Angst und Furchtreaktion als dominant

\section{Abstract \\ $\nabla$}

Common causes of mental disorders are revealed by transdiagnostic perspectives. In this context, based on the study of Epkins and Heckler, the present study investigates common risk factors in depressive and anxiety symptomatics of children and adolescents. In a systematic literature search between 2012 and 2014, 35 studies were identified which demonstrated 44 common risk factors in internalising symptomatics in childhood and adolescence. Female gender, the onset of puberty, compliance with chronic illness, increased stress experience of children and mothers, negative parenting, misuse or rejective experiences in peers are evident factors in the developmental psychopathology of depression and anxiety disorders in childhood or adolescence. These common risk factors suggest a similar treatment of these diseases.

und bei den Depressionen Schwierigkeiten mit der Regulation von Stimmungen und dem Antrieb im Alltag (Verlust an Aktivitäten, Interesse, Freude). Die Angaben zu den Lebenszeitprävalenzen der Kinder oder Jugendlichen mit Angststörungen und Depressionen schwanken stark und liegen zwischen 3 und 16\%; bei Jugendlichen ist die Prävalenz tendenziell höher als bei jüngeren Kindern [8 - 10]. Ursachen für die Entstehung dieser Störungen werden vielfach getrennt voneinander untersucht [11, 12], obwohl die hohe Komorbidität dieser Störungen hinlänglich bekannt ist; $28-56 \%$ aller Kinder oder Jugendlichen mit Depression weisen auch die Symptomatik einer Angststörung auf $[13,14]$. Es bleibt daher unklar, ob Angststörungen und Depressionen eine ähnliche oder sogar dieselbe Ätiologie zugrunde liegt. 
Die Frage, inwieweit gemeinsame ätiologische Faktoren die Entstehung und den Verlauf von Angststörungen und Depressionen bestimmen, soll in der vorliegenden Studie geklärt werden.

\section{Die transdiagnostische Perspektive}

In der Transdiagnostik wird davon ausgegangen, dass psychopathologisch bei allen psychischen Erkrankungen Defizite im prosozialen Verhalten und Defizite in kognitiven, emotionalen und physiologischen Bereichen vorliegen können [15]. Störungsspezifische Ätiologie- oder Behandlungsmodelle reichen oft nicht aus, um der Komplexität psychischer Erkrankungen gerecht zu werden. Hohe Komorbiditätsraten psychischer Störungen oder eine Veränderung von Symptomen über längere Zeit (von einer Störung zu einer anderen) führten in der Vergangenheit dazu, dass ein Umdenken im klinischen Kontext erfolgte und störungsübergreifende Ansätze für die Erklärung und auch Behandlung von psychischen Erkrankungen entwickelt wurden [16]. Dieser transdiagnostische Ansatz berücksichtigt dabei unterschiedliche interpersonelle Prozesse wie Beziehungen zu Gleichaltrigen, Eltern-Kind-Beziehungen oder das Familiensystem. Zusätzlich integriert dieser Ansatz Umweltbedingungen wie das Schulklima, die Nachbarschaft, kulturelle Einflüsse oder sogar gesellschaftspolitische Grundsätze [15, 16]. Barlow und Kollegen [17] integrierten in einem universellen Behandlungssetting für Kinder und Jugendliche beispielsweise zunächst Aspekte wie die Förderung der Emotionsregulation, den Abbau von emotionaler Vermeidung oder Vermeidungsverhalten und die Umstrukturierung ungünstiger kognitiver Prozesse. Diese universelle Behandlung wurde im Verlauf der Zeit erweitert und auch die Wirkung von emotionalen Prozessen auf Kinder und Jugendliche, besonders im Kontext von emotionsauslösenden Situationen, wurde in die Behandlungssettings eingearbeitet [18]. Auch Southam-Garow und Kendall [19] betonen, dass in der Entwicklung von psychischen Störungen nicht hauptsächlich die dysfunktionalen kognitiven Mechanismen im Vordergrund stehen, sondern die Art der Emotion, die Kinder und Jugendliche mit bestimmten Situationen assoziieren. Compas und Kollegen [20] führen wiederum in diesem Kontext an, dass es nicht ausreicht, in der Ätiologie von psychischen Erkrankungen die Defizite in der Emotionsregulation zu fokussieren, sondern vermehrt Stress auslösende Situationen und die Art der Copingstrategien zu berücksichtigen sind. Diese Autorengruppe interpretiert die Emotionsregulation als eine Form von Copingstrategie, die in der Interpretation des transdiagnostischen Prozesses bei Defiziten zu verschiedenen psychischen Erkrankungen führen kann. Nolen-Hoeksma und Watkins [21] unterscheiden verschiedene Klassen von Risikofaktoren, unabhängig von der Art der psychischen Störung:

- proximale Faktoren wie neurobiologische/genetische Komponenten, die einen direkten Einfluss auf die Symptomatik ausüben (oder sie verursachen),

- distale Faktoren wie Persönlichkeitsmerkmale oder Umweltbedingungen, die nicht direkt auf eine psychische Erkrankung einwirken, sondern sie indirekt beeinflussen, und

- moderierende Mechanismen wie beispielsweise Intelligenz, die die Beziehung zwischen distalen Faktoren und der Symptomatik beeinflussen.

Bei der Ätiologie von Angststörungen und Depressionen gehen Dozois, Seeds und Collins [22] transdiagnostisch von vier zentralen Risikofaktoren aus:

1. negative Kognitionen,

2. Erziehungsaspekte,

3. Stress sowie

4. Temperament und Persönlichkeit.
Caspi und Kollegen [23] fokussieren diese Aspekte zu einem psychopathologischen Faktor, der generell im Kontext der Entwicklung von psychischen Erkrankungen wirkt. Die Autorengruppe untersuchte in einer Längsschnittstudie $(N=1037)$ die Wirkung von Risikofaktoren und konnte zunächst mithilfe von Strukturgleichungsmodellen herausfiltern, dass drei große Cluster an psychischen Störungen in der Wirkung aufzufinden sind: In der Symptomatik bildeten sich die Gruppen der externalisierenden, internalisierenden und weiteren Störungen heraus. Die von den Autoren als ,p-Faktor' bezeichnete Dimension der allgemeinen Ursachen beschreibt unabhängig von der Symptomatik eine Art ,generelle Intelligenz' und Coping, mit negativen Lebensereignissen umzugehen. Bei höheren Ausprägungen auf diesem p-Faktor sind eine stärkere Beeinträchtigung der Lebensqualität und eine massivere Psychopathologie vorhanden, die sich wie folgt charakterisieren lässt:

- Persönlichkeitsmerkmale wie eine geringe Verträglichkeit, starker Neurotizismus oder eine gering ausgeprägte Gewissenhaftigkeit,

- negative Erlebnisse und Überzeugungen wie stationäre Psychiatrieaufenthalte während der Kindheit, stark ausgeprägte Suizidgedanken, stärkere Einbindungen in die Gleichaltrigengruppe (statt der Familie) während der Pubertät, eine stark ausgeprägte Gewaltbereitschaft, Misshandlungserfahrungen während der Kindheit,

- eine niedrige Schichtzugehörigkeit, die assoziiert wird mit einem früh auftretenden Risiko, bereits während der Kindheit an einer psychischen Störung zu erkranken,

- Defizite in biologischen Komponenten, beispielsweise eine niedrige Intelligenz, Defizite in den Exekutivfunktionen, aber auch motorische Beeinträchtigungen,

- emotionale Dysregulation (z. B. geringe Selbstkontrolle und dysfunktionale Mechanismen [23]).

Ein interpersonelles Risikomodell am Beispiel der sozialen Ängstlichkeit und depressiver Symptomatik

In einer Übersichtsarbeit, die empirische Studien bis zum Jahr 2010 integriert, zeigen Epkins und Heckler [24] ein interpersonelles Risikofaktorenmodell für die soziale Ängstlichkeit und die depressive Smptomatik auf. $\bullet$ Tab. 1 berichtet zusammenfassend die Risikofaktoren, die basierend auf den Ergebnissen von Epkins und Heckler [24] bei diesen Störungen besonders häufig auftreten. Diese Faktoren geben einen spezifischeren Überblick zur sozialen Ängstlichkeit und der depressiven Symptomatik als der generelle p-Faktor nach Caspi, der, wie oben beschrieben, von bestimmten Persönlichkeitsmerkmalen wie einem starken Neurotizismus, von negativen Erlebnissen wie Psychiatrieaufenthalten, von einer starken Einbindung in Gleichaltrigengruppen in der Pubertät, von Defiziten in biologischen Komponenten oder von der Emotionsregulation gekennzeichnet ist [23]. Es wird bei Epkins und Heckler [24] aber deutlich, dass individuelle Merkmale wie die Verhaltenshemmung oder auch Erfahrungen familiärer/elterlicher Zurückweisungen spezifisch die Gefahr für Depressionen oder soziale Ängstlichkeit erhöhen können [24]. Gleichzeitig ist auffällig, dass es nicht nur die nach Caspi [23] postulierte starke Einbindung in die Gleichaltrigengruppe während der Pubertät sein kann, die psychopathologisch wirkt. Für die depressive Symptomatik und die soziale Ängstlichkeit sind es besonders die sozialen Zurückweisungen bis hin zu Bullying-Erfahrungen mit Gleichaltrigen, die bei Kindern und Jugendlichen zu den internalisierenden Symptomen führen können. An dieser Stelle soll hervorgehoben werden, dass die Grenzen der Symptomatik fließend sein können, bis dann weiter- 
Tab. 1 Zusammenfassung der Risikofaktoren für soziale Ängstlichkeit und Depression, modifiziert nach Epkins und Heckler [24].

\begin{tabular}{|c|c|c|}
\hline & Soziale Ängstlichkeit & Depression \\
\hline \multirow[t]{4}{*}{ Biologisch } & Schüchternheit & $\begin{array}{l}\text { Geringe positive } \\
\text { Affektivität }\end{array}$ \\
\hline & Verhaltenshemmung & Verhaltenshemmung \\
\hline & Genetische Labilität & Genetische Labilität \\
\hline & $\begin{array}{l}\text { Ausgeprägte negative } \\
\text { Affektivität }\end{array}$ & $\begin{array}{l}\text { Ausgeprägte negative } \\
\text { Affektivität }\end{array}$ \\
\hline \multirow[t]{6}{*}{ Familiär } & Eltern mit Angststörung & Eltern mit Angststörung \\
\hline & Eltern mit Depressionen & $\begin{array}{l}\text { Eltern oder Großeltern } \\
\text { mit Depressionen }\end{array}$ \\
\hline & Unsichere Bindungen & Unsichere Bindungen \\
\hline & Elterliche Zurückweisung & $\begin{array}{l}\text { Familienkonflikte wie } \\
\text { Beziehungsprobleme } \\
\text { der Eltern }\end{array}$ \\
\hline & $\begin{array}{l}\text { Geringe elterliche } \\
\text { Zuneigung }\end{array}$ & $\begin{array}{l}\text { Geringe elterliche } \\
\text { Zuneigung }\end{array}$ \\
\hline & Elterliche Überbehütung & \\
\hline \multirow[t]{7}{*}{ Sozial } & $\begin{array}{l}\text { Soziale Isolation/ } \\
\text { Einsamkeit }\end{array}$ & Soziale Ängstlichkeit \\
\hline & $\begin{array}{l}\text { Ausschluss aus der } \\
\text { Peer-Group }\end{array}$ & Einsamkeit \\
\hline & Mobbingopfer & Mobbingopfer \\
\hline & $\begin{array}{l}\text { Geringe Intimität } \\
\text { in Freundschaften }\end{array}$ & $\begin{array}{l}\text { Geringe soziale } \\
\text { Akzeptanz }\end{array}$ \\
\hline & $\begin{array}{l}\text { Keine positiven Freund- } \\
\text { schaftserfahrungen }\end{array}$ & $\begin{array}{l}\text { Keine positiven Freund- } \\
\text { schaftserfahrungen }\end{array}$ \\
\hline & Geringe soziale & Geringe soziale \\
\hline & Kompetenzen & Kompetenzen \\
\hline
\end{tabular}

führend die Diagnose der Depression bei Kindern oder Jugendlichen vorliegen kann. Die Risikofaktoren beeinflussen allerdings bereits auch vor dem Auftreten aller Symptome im Sinne einer klinischen Diagnose die pathologische Entwicklung. Aufgrund dessen fokussieren Epkins und Heckler in ihrer Übersichtsarbeit nicht nur das reine Störungsbild der sozialen Phobie oder Depression, sondern auch die Vorläufersymptomatik, die dann zur psychischen Erkrankung führen kann [24].

Basierend auf den Ergebnissen von Epkins und Heckler [24] möchte die vorliegende Studie eine Übersicht zum derzeitigen Forschungsstand zu den gemeinsamen Risikofaktoren für die depressive und ängstliche Symptomatik im Kindes- und Jugendalter geben. Es soll aus transdiagnostischer Perspektive untersucht werden, ob gemeinsame Risikofaktoren im Kindes- und Jugendalter nicht nur für die soziale Ängstlichkeit und die depressive Symptomatik vorliegen, sondern für alle Angststörungen (z.B. auch Generalisierte Angststörung, Trennungsängste) oder ausgeprägt ängstliche Symptomatik und die depressive Symptomatik. Auch diese Studie fokussiert, basierend auf dem Vorgehen von Epkins und Heckler [24], nicht nur die klinisch-psychiatrischen Diagnosen, sondern untersucht den Einfluss der Risikofaktoren bereits bei ausgeprägter ängstlicher oder depressiver Symptomatik bei Kindern und Jugendlichen.

\section{Methode}

\section{Literaturrecherche}

Mithilfe von manueller Literaturrecherche und systematischer Literaturrecherche auf den Datenbanken Pubmed, Psychinfo und Web of Science gibt diese Studie eine aktuelle Übersicht über die gemeinsamen Risikofaktoren von ängstlicher oder depressiver Symptomatik im Kindes- und Jugendalter. Methodisch werden die Standards für die Konzeption von systematischen Reviews berücksichtigt [25]. Um alle Studien zu Risikofaktoren von ängstlicher oder depressiver Symptomatik herauszufiltern, wurden folgende Schlagwörter in der Literaturrecherche verwendet:

- Depressive Symptomatik: [depression OR affective disorders OR bipolar disorders OR internalizing OR emotional disorders OR melancholy] AND

- Angstsymptomatik: [anxiety disorders OR anxiety OR fear OR obsessive-compulsive disorders OR social anxiety OR generalized anxiety] AND

- Kindheit: [children OR child OR adolescents OR youth OR childhood] AND

- Gemeinsame Risikofaktoren: [risk factors OR causes OR etiology].

\section{Studienauswahl}

Für die Analyse der gemeinsamen Risikofaktoren wurden Einund Ausschlusskriterien für die Studienauswahl formuliert. Die Studien müssen basierend auf folgenden Kriterien durchgeführt worden sein, um sie in das vorliegende Review aufzunehmen:

- Studien mit Kindern und Jugendlichen bis zu 20 Jahren wurden berücksichtigt, da die Festlegung des Erwachsenenalters sich international unterscheiden kann (zwischen 18 und 21 Jahren).

- Die Studien untersuchten Ursachen von ängstlicher oder depressiver Symptomatik im Kindes- und Jugendalter. Studien, die nur Komorbiditäten einbezogen, wurden ausgeschlossen. Um die transdiagnostische Perspektive zu berücksichtigen, wurden nur Studien integriert, die Risikofaktoren bei beiden Störungsbildern oder Symptomgruppen untersuchten und nicht für jede Störung oder Symptomatik einzeln.

- Die psychischen Störungen wurden mit standardisierter Methodik (Fragebögen, Interviews) erfasst.

- Die Depressionen oder Angststörungen der Kinder oder Jugendlichen sind klassifiziert nach ICD-10 (bspw. F 3, F 4 oder F 93) [26], DSM-IV [27] oder DSM-5 [7] oder es liegen erhöhte Symptomausprägungen (Ängstlichkeit, Depressivität) bei Kindern und Jugendlichen vor.

- Es können auch Komorbiditäten zu anderen Störungen vorhanden sein, aber es müssen in jedem Fall eine Depression oder eine Angststörung oder stark ausgeprägte internalisierende Symptome bei den untersuchten Kindern oder Jugendlichen vorliegen.

- Die Studien können zusammenfassend Risikofaktoren bei internalisierenden Störungen untersucht haben. Allerdings werden Studien ausgeschlossen, die unter der internalisierenden Symptomatik auch somatoforme/psychische Störungen, verursacht durch körperliche Faktoren oder Essstörungen, definieren, um ausschließlich die Wirkung von gemeinsamen Risikofaktoren auf Depression und Angststörungen aufzeigen zu können.

- Um einen aktuellen Überblick zu geben, wurden peer-reviewte Publikationen in deutscher oder englischer Sprache von Januar 2012 bis Juli 2014 berücksichtigt.

- Unterschiede müssen auf statistische Signifikanz überprüft worden sein und die Mindestanzahl der untersuchten Kinder oder Jugendlichen betrug $N=50$.

- Studien wurden ausgeschlossen, die ausschließlich Suizidalität untersuchen, wenn keine Angaben hinsichtlich einer vorhandenen Depression oder Angststörung vorliegen. 
- Studien zur Ursachenforschung über Persönlichkeitsstörungen (auch wenn es beispielsweise der ängstlich-vermeidende Typ ist) wurden ausgeschlossen.

\section{Ergebnisse}

$\nabla$

Die systematische Studienrecherche identifizierte zunächst 710 potenzielle Studien. Unter Berücksichtigung aller gelisteten Einund Ausschlusskriterien waren für die vorliegende Übersicht der gemeinsamen Risikofaktoren für Angststörungen und Depression im Kindes- und Jugendalter 35 Studien geeignet ( $\bullet$ Abb.1). Viele Studien (10) erfassten unterschiedliche Risikofaktoren für Angststörungen und Depression bei Kindern und Jugendlichen. Insgesamt zeigten 10 Studien biologische Risikofaktoren für Depression und Angststörungen bei Kindern und Jugendlichen auf, 11 Studien familiäre Risikofaktoren, 12 Studien soziale Risikofaktoren, 15 Studien psychische Risikofaktoren. Mithilfe der systematischen Recherche und aller eingeschlossenen Studien konnten somit 44 gemeinsame Risikofaktoren für ängstliche oder depressive Symptomatik im Kindes- und Jugendalter herausgefiltert werden. Davon waren fünf biologische Risikofaktoren, 16 familiäre, 11 soziale und 12 psychische Risikofaktoren. Mehrheitlich wurden Jugendliche zwischen 12 und 20 Jahren untersucht (in 19 Studien), 7 Studien berichten von Kindern und Jugendlichen über eine größere Altersgruppe ( 0 - 17 Jahre) und 9 Studien untersuchten jüngere Kinder vor Eintritt der Pubertät (<12 Jahre). Mehrheitlich wurden die internalisierenden Symptome von Kindern oder Jugendlichen selbst berichtet (bei 24 unterschiedlichen Risikofaktoren), 7 Risikofaktoren wurden herausgefiltert, indem der Selbstbericht mit Ergebnissen aus Lehrer-, Elternbefragung oder physiologischen Messungen kombiniert und validiert wurde, 11 Risikofaktoren durch reine Elternbefragung, ein Risikofaktor durch Elternbefragung und Speichelproben der Kinder und ein Risikofaktor durch Eltern- und Lehrerbefragung. Im Folgenden werden die Ergebnisse nach Art der Risikofaktoren (biologisch, familiär, sozial) gruppiert und für jeden Bereich einzeln aufgeführt. Da die Einteilung in drei Risikofaktorengruppen für eine angemessene Einordnung der aufgefundenen Ergebnisse nicht ausreicht, wurde eine vierte Kategorie der psychischen Risikofaktoren hinzugefügt. Diese Kategorie integriert alle individuellen Faktoren, die eher kognitive Komponenten (wie z. B. das Selbstbild der Kinder und Jugendlichen) beschreiben.

\section{Biologische/Genetische Risikofaktoren}

Die aktuelle Literaturrecherche konnte fünf unterschiedliche biologische Risikofaktoren für ängstliche oder depressive Symptomatik bei Kindern und Jugendlichen aufzeigen (vgl.๑ Tab. 2). Im Verlauf der Kindheit und Adoleszenz kann festgestellt werden, dass die Gefahr für eine pathologische Entwicklung von Depression oder Angststörungen mit zunehmendem Alter steigt [28, 29]. Zudem wird in fünf Studien mit sehr unterschiedlichen Stichproben (z. B. klinische Stichproben, Sonderschulen oder Allgemeinbevölkerung [30 - 32]) das weibliche Geschlecht als Risikofaktor für Depression und Angststörungen im Kindes- und Jugendalter herausgefiltert [30-34]. Genetische Untersuchungen, die Kinder mit der langen Form des Serotonintransporters mit Kindern mit der kurzen Form verglichen, konnten wiederum aufzeigen, dass Kinder bereits im Alter zwischen 3 und 5 Jahren eine erhöhte Gefahr für internalisierende Probleme aufwiesen, wenn bei ihnen die lange Form des Serotonintransporters festgestellt werden konnte [35]. Gleichzeitig zeigten weitere neurologische Untersuchungen, dass Traumata in der frühen Kindheit die funktionale Verknüpfung zwischen der Amygdala und dem Hippocampus verringern können [36]. Dieses geringe Funktionsniveau konnte als Risikofaktor für Depression und Angststörungen bei Jugendlichen nachgewiesen werden [36]. Eine pakistanische Studie mit einer klinischen Stichprobe stellte wiederum eine erhöhte Prävalenz von Depression und Angststörungen bei Kindern und Jugendlichen fest, wenn sie körperliche Symptome ohne geklärte Ursache aufwiesen (wie beispielsweise organische Schmerzen [37]; vgl.๑ Tab. 2).

\section{Familiäre Risikofaktoren}

Bei den insgesamt 16 verschiedenen familiären Risikofaktoren in - Tab. 3 fällt besonders die Bedeutung der Mütter für die Ent-

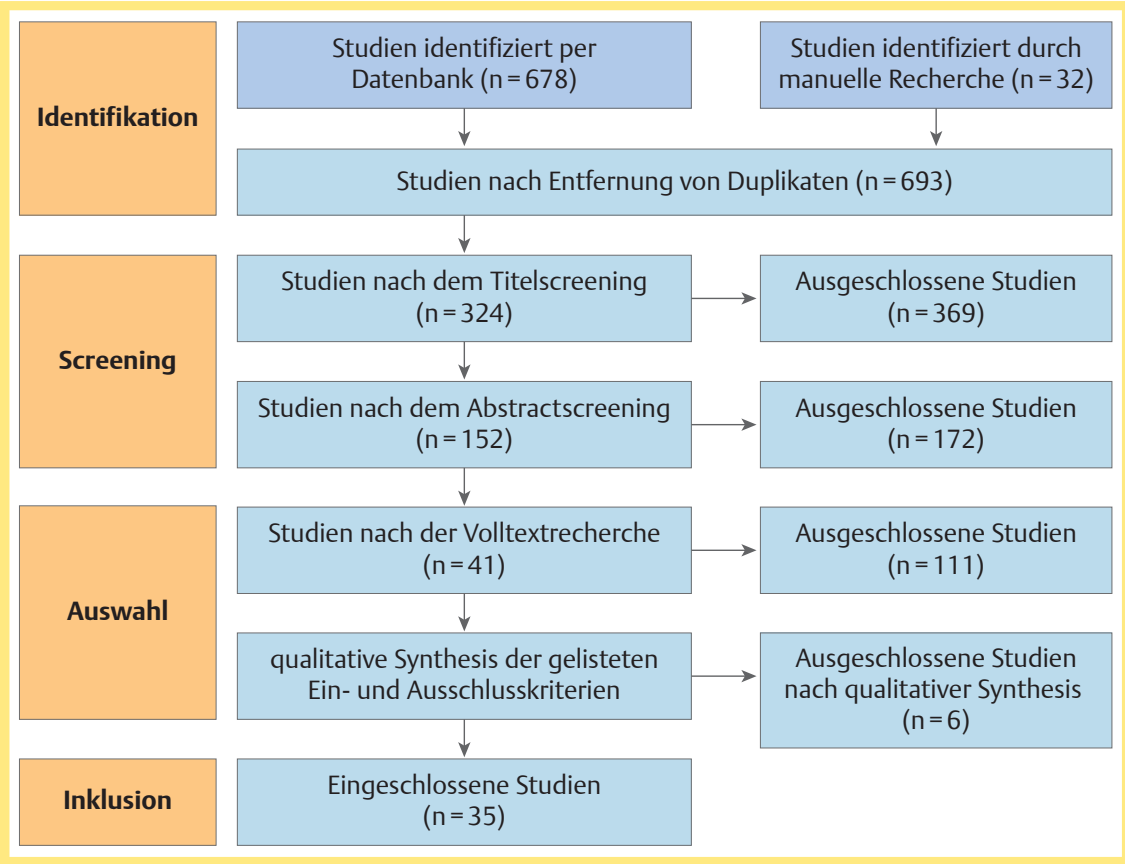

Abb.1 Flowchart der Studienselektion 
Tab. 2 Gemeinsame biologische/genetische Risikofaktoren für Angststörungen und Depression im Kindes- und Jugendalter.

\begin{tabular}{|c|c|c|c|c|c|c|}
\hline $\begin{array}{l}\text { Risikofaktoren } \\
\left(\mathrm{OR}^{1}\right)\end{array}$ & Autoren & Population & Charakteristika der Stichprobe & Alter & $\begin{array}{l}\text { Messinstru- } \\
\text { mente }^{2,3}\end{array}$ & $\begin{array}{l}\text { Art der } \\
\text { Messung }\end{array}$ \\
\hline \multirow[t]{2}{*}{$\begin{array}{l}\text { Zunehmendes } \\
\text { Alter: Adoles- } \\
\text { zenz }(2,05)\end{array}$} & $\begin{array}{l}\text { O-Dell \& } \\
\text { DuPaul } \\
(2012)[28]\end{array}$ & $\begin{array}{l}779 \text { Kinder } \\
\text { und } \\
\text { Jugendliche }\end{array}$ & $\begin{array}{l}\text { Klinische Stichprobe mit Kindern und } \\
\text { Jugendlichen mit Diabetes mellitus } \\
\text { zur Untersuchung emotionaler Pro- } \\
\text { bleme. }\end{array}$ & $\begin{array}{l}0-17 \text { Jahre } \\
(M=12,7)\end{array}$ & NS-CSHCN & $\begin{array}{l}\text { Telefonische } \\
\text { Elternbefra- } \\
\text { gung }\end{array}$ \\
\hline & $\begin{array}{l}\text { Skrove et al. } \\
\text { (2013) [29] }\end{array}$ & $\begin{array}{l}7639 \\
\text { Jugendliche }\end{array}$ & $\begin{array}{l}\text { Population aus der ,Nord Trondelag } \\
\text { Health Study، (Young-Hunt-Study 3) } \\
\text { in Norwegen }\end{array}$ & $\begin{array}{l}13-18 \\
\text { Jahre }\end{array}$ & SCL-5; HUNT & Selbstbericht \\
\hline \multirow[t]{5}{*}{$\begin{array}{l}\text { Weibliches } \\
\text { Geschlecht }\end{array}$} & $\begin{array}{l}\text { De Jong et al. } \\
\text { (2012) [30] }\end{array}$ & $\begin{array}{l}1806 \\
\text { Jugendliche }\end{array}$ & $\begin{array}{l}\text { Schüler der ersten beiden Sekundar- } \\
\text { schulklassen aus den Niederlanden }\end{array}$ & $\begin{array}{l}M=13,6 \\
\text { Jahre }\end{array}$ & RCADS; RSES & $\begin{array}{l}\text { Elternbefra- } \\
\text { gung und } \\
\text { Selbstbericht }\end{array}$ \\
\hline & $\begin{array}{l}\text { Slopen et al. } \\
\text { (2012) [31] }\end{array}$ & $\begin{array}{l}2345 \\
\text { Jugendliche }\end{array}$ & Schüler aus Chicago, USA & $\begin{array}{l}\mathrm{T} 1: M=14 \\
\mathrm{~T} 2: M=16,5 \\
\text { Jahre }\end{array}$ & DISC-IV & Selbstbericht \\
\hline & $\begin{array}{l}\text { Bean } \\
(2012)[32]\end{array}$ & $\begin{array}{l}126 \\
\text { Jugendliche }\end{array}$ & $\begin{array}{l}\text { Kinder in Sonderschulen mit afrikani- } \\
\text { scher Ethnie, rekrutiert im regionalen } \\
\text { medizinischen Zentrum in Tennes- } \\
\text { see, USA }\end{array}$ & 12 Jahre & $\mathrm{CBCL}$ & $\begin{array}{l}\text { Eltern- und } \\
\text { Lehrerbefra- } \\
\text { gung }\end{array}$ \\
\hline & $\begin{array}{l}\text { Ghoul et al. } \\
\text { (2013) [33] }\end{array}$ & $\begin{array}{l}716 \\
\text { Jugendliche }\end{array}$ & $\begin{array}{l}\text { Schüler aus einer Sekundarschule } \\
\text { (high school) im Nordosten der USA }\end{array}$ & $\begin{array}{l}M=16,0 \\
\text { Jahre }\end{array}$ & RCADS; SWCQ & Selbstbericht \\
\hline & $\begin{array}{l}\text { Glasheen } \\
\text { et al. (2013) } \\
\text { [34] }\end{array}$ & 577 Mütter & $\begin{array}{l}\text { Mütter, die in der Schwangerschaft in } \\
\text { einer Klinik rekrutiert wurden; im } \\
\text { Rahmen des Maternal Health Practi- } \\
\text { ces and Child Development Project' } \\
\text { und dem ,Childhood Abuse as a Pre- } \\
\text { dictor of Adolescent Alcohol Project" } \\
\text { in den USA }\end{array}$ & $\begin{array}{l}3 \text { - } 16 \text { Jahre } \\
\text { (mehrere } \\
\text { Messzeit- } \\
\text { punkte) }\end{array}$ & $\begin{array}{l}\text { CES-D; STPI; } \\
\text { DISC-IV }\end{array}$ & $\begin{array}{l}\text { Mütterbefra- } \\
\text { gung und } \\
\text { Selbstbericht }\end{array}$ \\
\hline $\begin{array}{l}\text { Lange Form des } \\
\text { Serotonin- } \\
\text { Transporters(5 } \\
\text { HTTLPR) }\end{array}$ & $\begin{array}{l}\text { Lavigne et al. } \\
\text { (2013) [35] }\end{array}$ & 175 Kinder & $\begin{array}{l}\text { Stichprobe mit Vorschülern rekru- } \\
\text { tiert aus } 23 \text { pädiatrischen Praxen in } \\
\text { Cook County, Illinois, USA }\end{array}$ & 3 - 5 Jahre & $\begin{array}{l}\text { DISC-YC; CSI; } \\
\text { ECBI; Speichel- } \\
\text { probe }\end{array}$ & $\begin{array}{l}\text { Elternbefra- } \\
\text { gung und } \\
\text { Entnahme der } \\
\text { Speichelprobe } \\
\text { per Hausbe- } \\
\text { such }\end{array}$ \\
\hline $\begin{array}{l}\text { Geringes Funk- } \\
\text { tionsniveau zwi- } \\
\text { schen Amygdala } \\
\text { und Hippocam- } \\
\text { pus, verursacht } \\
\text { durch Traumata } \\
\text { in der Kindheit }\end{array}$ & $\begin{array}{l}\text { Herringa et al. } \\
\text { (2013) [36] }\end{array}$ & $\begin{array}{l}64 \\
\text { Jugendliche }\end{array}$ & $\begin{array}{l}\text { Stichprobe aus der, Wisconsin Study } \\
\text { of Families and Work' in den USA }\end{array}$ & $\begin{array}{l}M=18,8 \\
\text { Jahre }\end{array}$ & $\begin{array}{l}\text { CTQ, HBQ; } \\
\text { FMRT-Messun- } \\
\text { gen }\end{array}$ & $\begin{array}{l}\text { Selbstbericht } \\
\text { und physiologi- } \\
\text { sche Gehirn- } \\
\text { Messungen }\end{array}$ \\
\hline $\begin{array}{l}\text { Ungeklärte } \\
\text { medizinische } \\
\text { Symptome }\end{array}$ & $\begin{array}{l}\text { Imran et al. } \\
\text { (2014) [37] }\end{array}$ & $\begin{array}{l}62 \text { Kinder und } \\
\text { Jugendliche }\end{array}$ & $\begin{array}{l}\text { Stichprobe aus einer Kinder- und Ju- } \\
\text { gendpsychiatrie in Lahore, Pakistan }\end{array}$ & $\begin{array}{l}M=11,7 \\
\text { Jahre }\end{array}$ & $\begin{array}{l}\text { CSI-24; SMFQ; } \\
\text { SCAS }\end{array}$ & Selbstbericht \\
\hline
\end{tabular}

NS-CSHCN: Nationwide telephone survey of non-internalized children (Child and Adolescent Health Measurement Initiative, 2007); RCADS: Revised Child Anxiety and Depression Scale (Chorpita et al, 2005); RSES: Rosenberg Self-Esteem Scale (Rosenberg, 1965); DISC-IV: Diagnostic Interview Schedule for Children IV (Shaffer et al., 2000) CBCL: Child and Behavior Checklist (Achenbach, 1997); SCL-5: Hopkins Symptom Checklist (Tambs et al 1993); HUNT: Originalfragebogen der Young-HUNT-3-Study; DISC-YC: Diagnostic Interview Schedule for Children Parent Scale Young Child; CSI: Child Symptom Inventory (Gadow \& Sprafkin, 2002); ECBI: Eyberg Child Behavior Inventory (Burns \& Patterson, 1990); SWCQ: Self-Worth Contingency Questionnaire (Burwell \& Shirk, 2006); CES-D: Centre for Epidemiological Studies Depression Scale (Radloff, 1977); STPI: Spielberger's State-Trait Anxiety Personality Inventory (Spielberger, 1979); CTQ: Childhood Trauma Questionnaire (Bernstein et al., 2003); HBQ: Health and Behavior Questionnaire (Essex et al., 2002); CSI-24: Children's Somatisation Inventory (Walker et al., 2009); SMFQ: Short Mood and Feelings-Questionnaire (Angold et al., 1995); SCAS: Spencer Children's Anxiety Scale (Spence et al., 2003); OR: Odds Ratio.

${ }^{1}$ Wenn in den Studien angegeben.

2 In allen Studien werden nur die Verfahren angegeben, die für die Untersuchung der Risikofaktoren relevant sind.

${ }^{3}$ Für die Messverfahren werden im Folgenden die Namen und mindestens der Erstautor genannt; die Quellen der Messverfahren sind unter folgendem Link abrufbar: www.zkpr.unibremen.de.

wicklung von ängstlicher oder depressiver Symptomatik bei Kindern und Jugendlichen auf. Es sind sieben Risikofaktoren aus den eingeschlossenen Studien ersichtlich, die den Einfluss der Mütter unterstrichen. So wirkte sich eine schlechte psychische Verfassung der Mütter wie Depression [38], Essstörungen [39] oder Substanzmissbrauch [43] negativ auf Kinder oder Jugendliche aus und erhöhte das Risiko für die Entwicklung von ängstlicher oder depressiver Symptomatik. Dieser Sachverhalt galt ebenfalls für Kinder, deren Mütter geringe Kompetenzen im Umgang mit Emotionen [41] oder eine schlechte Selbstbeherrschung aufwie- sen [32]. Im Rahmen einer amerikanischen Längsschnittstudie, bei der Kinder vom Kleinkindalter bis zur Pubertät zu verschiedenen Messzeitpunkten untersucht wurden, konnte aufgezeigt werden, dass bei geringer sozialer Unterstützung durch Mütter deren Kinder ebenfalls vermehrt eine ängstliche oder depressive Symptomatik aufwiesen [44].

Die Familiensituation wirkte sich ebenfalls signifikant auf die psychische Verfassung von Kindern und Jugendlichen aus. So zeigten Kinder oder Jugendliche mit einem alleinerziehenden Elternteil [43] oder ohne ältere Geschwister [43] vermehrt interna- 
Tab. 3 Gemeinsame familiäre Risikofaktoren für Angststörungen und Depression im Kindes- und Jugendalter.

\begin{tabular}{|c|c|c|c|c|c|c|}
\hline Risikofaktoren (OR ${ }^{1}$ ) & Autoren & Population & Charakteristika der Stichprobe & Alter & $\begin{array}{l}\text { Messinstru- } \\
\text { mente }^{2,3}\end{array}$ & Art der Messung \\
\hline $\begin{array}{l}\text { Depression der } \\
\text { Mütter }\end{array}$ & $\begin{array}{l}\text { Mendes et al. } \\
\text { (2013) [38] }\end{array}$ & 120 Kinder & $\begin{array}{l}\text { Stichprobe rekrutiert von Frauen } \\
\text { (Müttern) aus der Gesundheitsver- } \\
\text { sorgung in Minas Gerais, Brasilien }\end{array}$ & 6-12 Jahre & $\begin{array}{l}\text { DAWBA, } \\
\text { SCID-IV }\end{array}$ & Elternbefragung \\
\hline $\begin{array}{l}\text { Essstörungen der } \\
\text { Mütter }\end{array}$ & $\begin{array}{l}\text { Nadia et al. } \\
\text { (2014) [39] }\end{array}$ & 9787 Kinder & $\begin{array}{l}\text { Stichprobe aus der, Avon longitu- } \\
\text { dinal study of parents and children } \\
\text { (ALSPAC)' in England (UK) }\end{array}$ & $\begin{array}{l}\text { Messzeitpunkte: } \\
7,10 \text { und } 13 \text { Jahre }\end{array}$ & DAWBA & Elternbefragung \\
\hline $\begin{array}{l}\text { Ungesunde Ernäh- } \\
\text { rung der Kinder }\end{array}$ & $\begin{array}{l}\text { Jacka et al. } \\
\text { (2013) [40] }\end{array}$ & 23.050 Kinder & $\begin{array}{l}\text { Stichprobe rekrutiert im Rahmen } \\
\text { der Norwegian Mother and Child } \\
\text { Cohort Study (MoBa) }\end{array}$ & $\begin{array}{l}\text { Messzeitpunkte: } 6 \\
\text { Monate, 1,5, } 3 \text { und } \\
5 \text { Jahre }\end{array}$ & $\mathrm{CBCL}$ & Elternbefragung \\
\hline $\begin{array}{l}\text { Negative Emotionali- } \\
\text { tät der Mütter }\end{array}$ & $\begin{array}{l}\text { Mills et al. } \\
\text { (2012) [41] }\end{array}$ & 523 Kinder & $\begin{array}{l}\text { Stichprobe aus dem Concordia } \\
\text { Longitudinal Risk-Project und der } \\
\text { Daycare and Preschool Adjustment } \\
\text { Study aus Kanada }\end{array}$ & 2-6 Jahre & CBQ, EAS & Elternbefragung \\
\hline $\begin{array}{l}\text { Familiärer sozio-öko- } \\
\text { nomischer Stress }\end{array}$ & $\begin{array}{l}\text { Mills et al. } \\
(2012)[41]\end{array}$ & s.o. & & & & \\
\hline $\begin{array}{l}\text { Erziehungsverhalten } \\
\text { mit viel Kritik gegen- } \\
\text { über dem Kind }\end{array}$ & $\begin{array}{l}\text { Mills et al. } \\
\text { (2012) [41] }\end{array}$ & s.o. & & & & \\
\hline $\begin{array}{l}\text { Starke Eltern-Kind- } \\
\text { Konflikte }\end{array}$ & $\begin{array}{l}\text { Huang et al. } \\
\text { (2012) [42] }\end{array}$ & 101 Kinder & $\begin{array}{l}\text { Stichprobe mit asiatischer } \\
\text { Ethnie rekrutiert aus } \\
\text { Kindergärten in den USA }\end{array}$ & $\mathrm{M}=4,2$ Jahre & PAS, BASC-2 & Elternbefragung \\
\hline $\begin{array}{l}\text { Erziehungsverhalten } \\
\text { mit der Forderung } \\
\text { nach strenger } \\
\text { Disziplin }\end{array}$ & $\begin{array}{l}\text { Bayer et al. } \\
\text { (2012) [43] }\end{array}$ & 733 Kinder & $\begin{array}{l}\text { Stichprobe aus der Gesundheits- } \\
\text { versorgung in Australien mit meh- } \\
\text { reren Messzeitpunkten }\end{array}$ & $\begin{array}{l}\text { Messzeitpunkte: } 7 \\
\text { Monate, } 1 \text { Jahr, } 1,5 \\
\text { Jahre, } 2 \text { Jahre, } 3 \text { Jahre } \\
\text { und } 5 \text { Jahre }\end{array}$ & $\mathrm{CBCL}$ & Elternbefragung \\
\hline $\begin{array}{l}\text { Geringe soziale } \\
\text { Unterstützung } \\
\text { durch die Mutter }\end{array}$ & $\begin{array}{l}\text { Glasheen et al. } \\
\text { (2013) [44] }\end{array}$ & 577 Mütter & $\begin{array}{l}\text { Mütter, die während der Schwan- } \\
\text { gerschaft in einer Klinik rekrutiert } \\
\text { wurden; im Rahmen des, Maternal } \\
\text { Health Practices and Child Deve- } \\
\text { lopment Project' und dem Child- } \\
\text { hood Abuse as a Predictor of Ado- } \\
\text { lescent Alcohol Project‘ in den USA }\end{array}$ & $\begin{array}{l}\text { 3- } 16 \text { Jahre (mehrere } \\
\text { Messzeitpunkte) }\end{array}$ & $\begin{array}{l}\text { CES-D; STPI; } \\
\text { DISC-IV }\end{array}$ & $\begin{array}{l}\text { Mütterbefragung } \\
\text { und Selbstbericht }\end{array}$ \\
\hline Mütterlicher Stress & $\begin{array}{l}\text { Bayer et al. } \\
\text { (2012) [43] }\end{array}$ & s.o. & & & & \\
\hline $\begin{array}{l}\text { Alleinerziehender } \\
\text { Elternteil }\end{array}$ & $\begin{array}{l}\text { Bayer et al. } \\
\text { (2012) [43] }\end{array}$ & s.o. & & & & \\
\hline $\begin{array}{l}\text { Substanzmissbrauch } \\
\text { der Mütter }\end{array}$ & $\begin{array}{l}\text { Bayer et al. } \\
\text { (2012) [43] }\end{array}$ & s.o. & & & & \\
\hline $\begin{array}{l}\text { Ohne ältere } \\
\text { Geschwister }\end{array}$ & $\begin{array}{l}\text { Bayer et al. } \\
\text { (2012) [43] }\end{array}$ & s.o. & & & & \\
\hline $\begin{array}{l}\text { Schlechte mütterli- } \\
\text { che Selbstbeherr- } \\
\text { schung }\end{array}$ & $\begin{array}{l}\text { Bean } \\
(2012) \text { [32] }\end{array}$ & 126 Jugendliche & $\begin{array}{l}\text { Kinder in Sonderschulen mit afri- } \\
\text { kanischer Ethnie, rekrutiert im re- } \\
\text { gionalen medizinischen Zentrum } \\
\text { in Tennessee, USA }\end{array}$ & 12 Jahre & $\mathrm{CBCL}$ & $\begin{array}{l}\text { Eltern- und Leh- } \\
\text { rerbefragung }\end{array}$ \\
\hline $\begin{array}{l}\text { Emotionaler Miss- } \\
\text { brauch in der Familie }\end{array}$ & $\begin{array}{l}\text { Hamilton et al. } \\
\text { (2013) [45] }\end{array}$ & 225 Jugendliche & $\begin{array}{l}\text { Stichprobe rekrutiert aus öffentli- } \\
\text { chen und privaten Schulklassen } \\
\text { der Mittelstufe (ersten Sekundar- } \\
\text { schulklassen) in Philadelphia, USA }\end{array}$ & 12 und 13 Jahre & $\begin{array}{l}\text { CDI; MASC; } \\
\text { CTQ }\end{array}$ & Selbstbericht \\
\hline $\begin{array}{l}\text { Körperlicher Miss- } \\
\text { brauch/Nötigung } \\
\text { in der Familie }\end{array}$ & $\begin{array}{l}\text { Pajer et al. } \\
\text { (2014) [46] }\end{array}$ & 195 Jugendliche & $\begin{array}{l}\text { Stichprobe mit nichtschwangeren, } \\
\text { weiblichen Jugendlichen aus Ohio, } \\
\text { USA }\end{array}$ & $15-16$ Jahre & CAPI; DISC-IV & Selbstbericht \\
\hline
\end{tabular}

DAWBA: Development and Well-being Assessment for Children and Adolescents parental version (Goodman et al., 2000); SCID-IV: Structured Clinical Interview for DSM-III-R personality disorders (First et al., 1995); CBCL: Child and Behavior Checklist (Achenbach, 1997); CBQ: Children's Behavior Questionnaire (Rothbart et al., 2001); EAS: Temperament: Early developing personality traits (Buss \& Plomin, 1984); PAS: Preschool Anxiety Scale (Spence et al., 2001); BASC-2: Behavioral Assessment System for Children - $2^{\text {nd }}$ Edition (Reynolds et al., 2004); CDI: Children's Depression Inventory (Kovacs, 1992); Multidimensional Anxiety Scale for Children (March, 1997); CTQ: Childhood Trauma Questionnaire (Bernstein et al., 2003); CES-D: Centre for Epidemiological Studies Depression Scale (Radloff, 1977); STPI: Spielberger's State-Trait Anxiety Personality Inventory (Spielberger, 1979); CAPI: Child Abuse Potential Inventory (Milner, 1986); OR: Odds Ratio.

${ }^{1}$ Wenn in den Studien angegeben.

${ }^{2}$ In allen Studien werden nur die Verfahren angegeben, die für die Untersuchung der Risikofaktoren relevant sind.

${ }^{3}$ Für die Messverfahren werden im Folgenden die Namen und mindestens der Erstautor genannt; die Quellen der Messverfahren sind unter folgendem Link abrufbar: www.zkpr.unibremen.de. 
lisierende Symptome. Auch das Erziehungsverhalten von Eltern beeinflusste bedeutsam die Prävalenz von ängstlicher oder depressiver Symptomatik im Kindes- und Jugendalter. Bei Auftreten starker Eltern-Kind-Konflikte [42], einem Erziehungsverhalten mit vielen Kritikäußerungen dem Kind gegenüber [41] oder mit starken elterlichen Forderungen nach Disziplin [43] traten erhöht internalisierende Symptome bei Kindern und Jugendlichen auf. Empfanden Familien starke Belastungen durch sozioökonomische Probleme [41] oder lag eine ungesunde Ernährung der Kinder vor [40], entwickelten Kinder oder Jugendliche ebenfalls vermehrt Depression oder Angststörungen. Weitere familiäre Risikofaktoren für Jugendliche sind emotionaler [45] oder körperlicher Missbrauch [46] wie beispielsweise Schläge, Nötigungen oder Erpressungen durch Familienmitglieder (in der Regel Eltern, aber auch Geschwister; vgl. Tab.3).

\section{Soziale oder umweltbedingte Risikofaktoren}

Die elf sozialen Risikofaktoren, die durch die eingeschlossenen Studien aufgezeigt werden konnten, werden in 0 Tab. 4 berichtet. Dabei fallen besonders Stressbelastungen von Kindern und Jugendlichen auf, die zu einer erhöhten Prävalenz von Depression und Angststörungen führten. So zeigen die Studien von Espejo und Hammen [47], Barber und Kollegen [48], Uliaszek und Kollegen [49] sowie Michl und Kollegen [50], dass belastende Lebensereignisse und erhöhtes Stresserleben bei Kindern und Jugendlichen zu stark ausgeprägten internalisierenden Symptomen führten. Gleichzeitig wurde deutlich, dass eine andersartige ethnische Herkunft [42], Belastungen durch Akkulturationsstress in der Einwanderungsgesellschaft [51] oder Fluchterfahrungen ohne Begleitung durch Bezugspersonen [52] bei Kindern und Jugendlichen eine erhöhte Prävalenz von Depression und Angststörungen verursachten. Waren Kinder oder Jugendliche durch eine chronische Erkrankung belastet, die außerdem eine finanzielle Last für die Familie zur Folge hat, so entwickelten Kinder oder Jugendliche ebenfalls vermehrt Depression oder Angststörungen [28]. Stärker ausgeprägte internalisierende Symptome traten bei Jugendlichen (aus Chicago) auch dann auf, wenn sie in einer Nachbarschaft lebten, in der eine hohe Gewaltbereitschaft vorherrschte [53].

Ein weiteres Merkmal, das bei Kindern und Jugendlichen eine stark ausgeprägte Depression und Angststörungen verursachte, war die Qualität der sozialen Beziehungen. Wiesen Jugendliche beispielsweise eine geringe soziale Akzeptanz unter Gleichaltrigen auf und berichteten viele ablehnende Erfahrungen, zeigten sich vermehrt internalisierende Symptome [54, 55]. Eine weitere Studie von Ghoul und Kollegen [33] konnte diesen Sachverhalt der erhöhten Prävalenz von Depression und Angststörungen ebenfalls nachweisen, wenn Jugendliche Schikanierungen oder Mobbing durch die Gleichaltrigengruppe erlebt hatten. Lauer und Renk [54] unterstrichen zudem, dass sich das Auftreten von psychischen Problemen bei Personen im näheren sozialen Umfeld (Familie, Freunde) auf die psychische Verfassung von Jugendlichen auswirken kann. So zeigten Jugendliche, die den Umgang mit psychischen Problemen aus dem näheren Umfeld nicht kennengelernt hatten, vermehrt Depression oder Angststörungen (vgl. 0 Tab.4).

\section{Psychische Risikofaktoren}

Bei den in Tab.5 aufgeführten elf psychischen Risikofaktoren fallen besonders die Studien auf, die das Selbstbild von Kindern und Jugendlichen untersuchten. So zeigten Kinder und Jugendliche mit einem negativen Selbstbild wie einem geringen Selbstbe- wusstsein, wenig Optimismus, einer geringen Selbstwirksamkeit sowie einem negativen Körperbild von sich selbst vermehrt Depression und Angststörungen [30, 33, 57 - 59]. Zudem führte ein Temperament mit ausgeprägten Hemmungen oder einer starken Schüchternheit (Inhibition) zu einer erhöhten Prävalenz internalisierender Symptome bei Kindern und Jugendlichen [41, 62]. Trat bei Jugendlichen erhöhtes Grübeln und ,Sich-Sorgen-Machen' auf, war die Gefahr für die Entwicklung von Depression oder Angststörungen ebenfalls erhöht [50].

Der Umgang mit körperlichen Erkrankungen stellte ebenfalls einen Risikofaktor für Depression oder Angststörungen dar. So zeigten Myers und Kollegen [56] bei Kindern mit einer Leukämie-Diagnose, dass erhöhte Depression oder Angststörungen auftraten, wenn ein geringes Krankheitsverständnis der Leukämie-Erkrankung bei den Kindern vorhanden war. Gleichzeitig zeigten O'Dell und DuPaul [28], dass der Schweregrad einer chronischen Erkrankung ebenfalls die psychische Verfassung von Kindern oder Jugendlichen beeinflusst. Die Autoren analysierten eine Stichprobe mit Kindern und Jugendlichen mit Diabetes mellitus, bei denen Kinder und Jugendliche mit einem hohen Schweregrad der Erkrankung auch eine stärker ausgeprägte Depression und Angststörungen aufwiesen.

Eine chinesische Studie zeigte zudem, dass Kinder und Jugendliche mit Schlafstörungen vermehrt internalisierende Symptome zeigten [61]. Gleichzeitig führte eine geringe Aufmerksamkeitskontrolle zu einer stärker ausgeprägten Depression und Angststörungen [62, 63]; dasselbe galt für männliche Jugendliche, die schnell reizbar waren. Burke [60] untersuchte in diesem Kontext Jungen mit einer Störung des Sozialverhaltens. Bei denjenigen mit einer besonders ausgeprägten Reizbarkeit konnte ebenfalls eine erhöhte Prävalenz von Depression und Angststörungen nachgewiesen werden. Bei Insassen einer Jugendhaftanstalt in Kanada konnten außerdem bei denjenigen Jugendlichen stärker ausgeprägte internalisierende Symptome festgestellt werden, die besonders schwere Regelverstöße während der Haftzeit verübt hatten [64]. Gleichzeitig konnten Skrove und Kollegen [29] bei norwegischen Jugendlichen erhöhte Angststörungen und Depression feststellen, wenn sie Substanzen wie Alkohol, Nikotin oder illegale Drogen konsumierten (vgl.๑ Tab.5).

\section{Diskussion \\ $\nabla$}

Die vorliegende Studie konnte aus entwicklungspsychopathologischer Sicht die transdiagnostische Perspektive von Depression und Angststörungen unterstreichen, da durch die systematische Recherche viele gemeinsame Risikofaktoren für Depression und Angststörungen im Kindes- und Jugendalter aufgezeigt werden konnten. Besonders in Anbetracht des eher kurzen Zeitraums der ausgewählten Studien zwischen 2012 und 2014 überrascht diese große Menge der identifizierten gemeinsamen Risikofaktoren für Depression und Angststörungen. Zudem wird angesichts der vorliegenden Ergebnisse die Vielfalt der gemeinsamen Risikofaktoren für Depression und Angststörungen im Kindes- und Jugendalter deutlich, da noch mehr gemeinsame Aspekte bei Kindern und Jugendlichen einen pathologischen Zusammenhang dieser Störungsbilder aufzeigen als die eingangs erwähnte Übersicht von Epkins und Heckler [24] (vgl. $\bullet$ Tab. 1), beispielsweise die aufgezeigten unterschiedlichen familiären Faktoren. 
Tab. 4 Gemeinsame soziale/umweltbedingte Risikofaktoren für Angststörungen und Depression im Kindes- und Jugendalter.

\begin{tabular}{|c|c|c|c|c|c|c|}
\hline Risikofaktoren (OR'1) & Autoren & Population & Charakteristika der Stichprobe & Alter & $\begin{array}{l}\text { Messinstru- } \\
\text { mente }^{2,3}\end{array}$ & Art der Messung \\
\hline $\begin{array}{l}\text { Starke Kostenbelas- } \\
\text { tung durch chronische } \\
\text { Erkrankung }(1,92)\end{array}$ & $\begin{array}{l}\text { O-Dell \& DuPaul } \\
(2012)[28]\end{array}$ & $\begin{array}{l}779 \text { Kinder und } \\
\text { Jugendliche }\end{array}$ & $\begin{array}{l}\text { Klinische Stichprobe mit Kindern und } \\
\text { Jugendlichen mit Diabetes mellitus } \\
\text { zur Untersuchung emotionaler Pro- } \\
\text { bleme. }\end{array}$ & $\begin{array}{l}0-17 \text { Jahre } \\
(M=12,7)\end{array}$ & NS-CSHCN & $\begin{array}{l}\text { Telefonische El- } \\
\text { ternbefragung }\end{array}$ \\
\hline $\begin{array}{l}\text { Belastende Lebenser- } \\
\text { eignisse }(1,28)\end{array}$ & $\begin{array}{l}\text { Espejo \& Ham- } \\
\text { men (2012) [47] }\end{array}$ & 653 Jugendliche & $\begin{array}{l}\text { Stichprobe aus der Geburtskohorte } \\
\text { zwischen } 1981 \text { und } 1984 \text { einer Klinik } \\
\text { in Queensland, Australien }\end{array}$ & $\begin{array}{l}\text { T1: } 15 \text { Jahre } \\
\text { T2: } 20 \text { Jahre }\end{array}$ & $\begin{array}{l}\text { Mütter: SCID- } \\
\text { IV; Jugendliche: } \\
\text { K-SADS-E; SCID- } \\
\text { IV); Lebenser- } \\
\text { eignisse: LSI }\end{array}$ & $\begin{array}{l}\text { Mütterbefragung } \\
\text { und Selbstbericht }\end{array}$ \\
\hline $\begin{array}{l}\text { Erhöhtes Stresserle- } \\
\text { ben durch traumati- } \\
\text { sche Lebensereignisse }\end{array}$ & $\begin{array}{l}\text { Barber et al. } \\
\text { (2014) [48] }\end{array}$ & $\begin{array}{l}479 \text { Kinder und } \\
\text { Jugendliche }\end{array}$ & $\begin{array}{l}\text { Stichprobe mit traumatisierten Kin- } \\
\text { dern; rekrutiert aus kinder- und ju- } \\
\text { gendpsychiatrischen Versorgungsin- } \\
\text { stitutionen in den USA }\end{array}$ & 8 - 17 Jahre & $\begin{array}{l}\text { ASC-Kids; CDI; } \\
\text { RCMAS }\end{array}$ & Selbstbericht \\
\hline \multirow[t]{2}{*}{$\begin{array}{l}\text { Erhöhtes } \\
\text { Stresserleben }\end{array}$} & $\begin{array}{l}\text { Uliaszek et al. } \\
\text { (2012) [49] }\end{array}$ & 627 Jugendliche & $\begin{array}{l}\text { Rekrutiert im Rahmen des Youth- } \\
\text { Emotion-Projects an Schulen in } \\
\text { Regionen bei Chicago/Los Angeles, } \\
\text { USA }\end{array}$ & 15- 18 Jahre & SCID-IV; LSI & Selbstbericht \\
\hline & $\begin{array}{l}\text { Michl et al. } \\
\text { (2913) [50] }\end{array}$ & 1065 Jugendliche & $\begin{array}{l}\text { Schüler der Sekundarschulen (Grade } \\
6-8 \text { ) in Central Connecticut, USA }\end{array}$ & $M=12,2$ Jahre & $\begin{array}{l}\text { CDI; MASC; } \\
\text { CRSQ; LESC }\end{array}$ & Selbstbericht \\
\hline Ethnische Herkunft & $\begin{array}{l}\text { Huang et al. } \\
\text { (2012) [42] }\end{array}$ & 101 Kinder & $\begin{array}{l}\text { Stichprobe mit asiatischer Ethnie re- } \\
\text { krutiert aus Kindergärten in den USA }\end{array}$ & $M=4,2$ Jahre & PAS, BASC-2 & Elternbefragung \\
\hline Akkulturationsstress & $\begin{array}{l}\text { Sirin et al. } \\
\text { (2013) [51] }\end{array}$ & 286 Jugendliche & $\begin{array}{l}\text { Jugendliche mit Migrationshinter- } \\
\text { grund in der ersten oder zweiten Ge- } \\
\text { neration, rekrutiert an Schulen in New } \\
\text { York City, USA }\end{array}$ & $M=16,2$ Jahre & YSR & Selbstbericht \\
\hline $\begin{array}{l}\text { Fluchterfahrungen } \\
\text { ohne Begleitung }\end{array}$ & $\begin{array}{l}\text { Vervilet et al. } \\
\text { (2014) [52] }\end{array}$ & 307 Jugendliche & $\begin{array}{l}\text { Stichprobe mit unbegleiteten min- } \\
\text { derjährigen Flüchtlingen aus Norwe- } \\
\text { gen und Belgien }\end{array}$ & 15-18 Jahre & HSCL-37A & Selbstbericht \\
\hline $\begin{array}{l}\text { Gewalt in der Nach- } \\
\text { barschaft oder in wei- } \\
\text { teren Lebensberei- } \\
\text { chen (Familie, Schule) }\end{array}$ & $\begin{array}{l}\text { Slopen et al. } \\
\text { (2012) [53] }\end{array}$ & 2345 Jugendliche & Schüler aus Chicago, USA & $\begin{array}{l}T 1: M=14,0 \\
T 2: M=16,5 \\
\text { Jahre }\end{array}$ & DISC-IV & Selbstbericht \\
\hline $\begin{array}{l}\text { Erfahrungen von Schi- } \\
\text { kanierungen/ Mob- } \\
\text { bing durch Gleichalt- } \\
\text { rige }\end{array}$ & $\begin{array}{l}\text { Ghoul et al. } \\
\text { (2013) [33] }\end{array}$ & 716 Jugendliche & $\begin{array}{l}\text { Schüler aus einer Sekundarschule } \\
\text { (high school) im Nordosten der USA }\end{array}$ & $M=16,0$ Jahre & RCADS; SWCQ & Selbstbericht \\
\hline \multirow{2}{*}{$\begin{array}{l}\text { Geringe soziale Ak- } \\
\text { zeptanz/Ablehnung } \\
\text { durch Gleichaltrige }\end{array}$} & $\begin{array}{l}\text { Lauer \& Renk } \\
(2013)[54]\end{array}$ & 281 Jugendliche & $\begin{array}{l}\text { Schüler (57 \% Farbige) aus Florida, } \\
\text { USA }\end{array}$ & $M=15,6$ Jahre & TRF, YSR & $\begin{array}{l}\text { Selbstbericht und } \\
\text { Lehrerbefragung }\end{array}$ \\
\hline & $\begin{array}{l}\text { Fite et al. (2014) } \\
\text { [55] }\end{array}$ & 289 Jugendliche & $\begin{array}{l}\text { Stichprobe mit ausschließlich Jungen } \\
\text { aus der, ,Pittsburgh Youth Study“ in } \\
\text { den USA }\end{array}$ & $M=16,0$ Jahre & YSR & Selbstbericht \\
\hline $\begin{array}{l}\text { Wenig Erfahrungen } \\
\text { mit psychischen } \\
\text { Problemen von Perso- } \\
\text { nen in der näheren } \\
\text { Umgebung }\end{array}$ & $\begin{array}{l}\text { Lauer \& Renk } \\
\text { (2013) [54] }\end{array}$ & s.o. & & & & \\
\hline
\end{tabular}

NS-CSHCN: Nationwide telephone survey of non-internalized children (Child and Adolescent Health Measurement Initiative, 2007); SCID-IV: Structured Clinical Interview for DSMIII-R personality disorders (First et al., 1995); K-SADS: Schedule for affective disorders and schizophrenia for school-age children epidemiologic version-5 (Orvaschel, 1995); LSI: Stress Interview (Hammen et al., 1989); ASC-Kids: Acute Stress Checklist for Children (Kassam-Adams, 2006); CDI: Children’s Depression Inventory (Kovacs, 1992); RCMAS: Revised Children's Manifest Anxiety Scale (Reynolds \& Richmond, 1978); DISC-IV: Diagnostic Interview Schedule for Children IV (Shaffer et al., 2000); MASC: Multidimensional Anxiety Scale for Children (March, 1997); LESC: Life Events Scale for Children (Coddington, 1972); CRSQ: Children's Response Style Questionnaire (Abela et al., 2002); SWCQ: Self-Worth Contingency Questionnaire (Burwell \& Shirk, 2006); TRF: Teacher's Report Form (Achenbach \& Rescorla, 2001); YRF: Youth Self-Report (Achenbach \& Rescorla, 2001); HSCL-37A: Hopkins Symptom Checklist-37A (Bean et al., 2007); OR: Odds Ratio.

${ }^{1}$ Wenn in den Studien angegeben.

${ }^{2}$ In allen Studien werden nur die Verfahren angegeben, die für die Untersuchung der Risikofaktoren relevant sind.

${ }^{3}$ Für die Messverfahren werden im Folgenden die Namen und mindestens der Erstautor genannt; die Quellen der Messverfahren sind unter folgendem Link abrufbar: www.zkpr.unibremen.de.

\section{Zusammenfassung der Ergebnisse}

Biologische Risikofaktoren sind besonders das weibliche Geschlecht und das zunehmende Alter. Gleichzeitig stellt der Umgang mit somatischen Erkrankungen für Kinder und Jugendliche eine starke Belastung dar und erhöht die Gefahr für Depression und Angststörungen.
Unter den familiären Risikofaktoren haben die Mütter auf die Entwicklung ängstlicher oder depressiver Symptomatik bei Kindern und Jugendlichen einen starken Einfluss; bei psychischen Belastungen der Mütter, erhöhtem Stresserleben oder einer emotionalen Dysregulation der Mütter entwickeln Kinder oder Jugendliche vermehrt eine ängstliche oder depressive Symptomatik. 
Tab.5 Gemeinsame psychische Risikofaktoren für Angststörungen und Depression im Kindes- und Jugendalter.

\begin{tabular}{|c|c|c|c|c|c|c|}
\hline Risikofaktoren (OR $\left.{ }^{1}\right)$ & Autoren & Population & Charakteristika der Stichprobe & Alter & $\begin{array}{l}\text { Messinstru- } \\
\text { mente }^{2,3} \mathbf{m}\end{array}$ & $\begin{array}{l}\text { Art der } \\
\text { Messung }\end{array}$ \\
\hline $\begin{array}{l}\text { Ausgeprägter Schwe- } \\
\text { regrad einer chroni- } \\
\text { schen Erkrankung } \\
(3,40)\end{array}$ & $\begin{array}{l}\text { O-Dell \& DuPaul } \\
(2012)[28]\end{array}$ & $\begin{array}{l}779 \text { Kinder und } \\
\text { Jugendliche }\end{array}$ & $\begin{array}{l}\text { Klinische Stichprobe mit Kindern und } \\
\text { Jugendlichen mit Diabetes mellitus zur } \\
\text { Untersuchung emotionaler Probleme }\end{array}$ & $\begin{array}{l}0-17 \text { Jahre } \\
(M=12,7)\end{array}$ & NS-CSHCN & $\begin{array}{l}\text { Telefonische } \\
\text { Elternbefra- } \\
\text { gung }\end{array}$ \\
\hline $\begin{array}{l}\text { Krank- } \\
\text { heitsverständnis/ } \\
\text { Coping }\end{array}$ & $\begin{array}{l}\text { Myers et al. } \\
(2014)[56]\end{array}$ & 159 Kinder & $\begin{array}{l}\text { Stichprobe aus einer onkologischen Kli- } \\
\text { nik mit Kindern mit einer Leukämie- } \\
\text { Diagnose }\end{array}$ & 2-9 Jahre & BASC-2 & $\begin{array}{l}\text { Elternbefra- } \\
\text { gung }\end{array}$ \\
\hline \multirow[t]{2}{*}{$\begin{array}{l}\text { Gering ausgeprägtes } \\
\text { Selbstbewusstsein }\end{array}$} & $\begin{array}{l}\text { De Jong et al. } \\
\text { (2012) [30] }\end{array}$ & $\begin{array}{l}1806 \\
\text { Jugendliche }\end{array}$ & $\begin{array}{l}\text { Schüler der ersten beiden Sekundar- } \\
\text { schulklassen aus den Niederlanden }\end{array}$ & $M=13,6$ Jahre & RCADS; RSES & $\begin{array}{l}\text { Elternbefra- } \\
\text { gung und } \\
\text { Selbstbericht }\end{array}$ \\
\hline & $\begin{array}{l}\text { Van Tujil et al. } \\
\text { (2014) [57] }\end{array}$ & $\begin{array}{l}1641 \\
\text { Jugendliche }\end{array}$ & $\begin{array}{l}\text { Schüler der ersten beiden Sekundar- } \\
\text { schulklassen aus nördlichen Regionen } \\
\text { in den Niederlanden }\end{array}$ & $M=13,1$ Jahre & RCADS; RSES & Selbstbericht \\
\hline \multirow{2}{*}{$\begin{array}{l}\text { Negatives Selbstbild } \\
\text { (gering: Selbstbe- } \\
\text { wusstsein, Optimis- } \\
\text { mus oder Selbstwert) }\end{array}$} & $\begin{array}{l}\text { Keyfitz et al. } \\
\text { (2013) [58] }\end{array}$ & $\begin{array}{l}172 \text { Kinder und } \\
\text { Jugendliche }\end{array}$ & $\begin{array}{l}\text { Schüler (5.-8. Grad) aus dem Südwesten } \\
\text { Ontarios, Kanada }\end{array}$ & 9-14 Jahre & $\begin{array}{l}\text { SQC; MASC; } \\
\text { CDI }\end{array}$ & Selbstbericht \\
\hline & $\begin{array}{l}\text { Ghoul et al. } \\
\text { (2013) [33] }\end{array}$ & $\begin{array}{l}716 \\
\text { Jugendliche }\end{array}$ & $\begin{array}{l}\text { Schüler aus einer Sekundarschule (high } \\
\text { school) im Nordosten der USA }\end{array}$ & $M=16$ Jahre & RCADS; SWCQ & Selbstbericht \\
\hline $\begin{array}{l}\text { Negatives Selbstbild } \\
\text { vom eigenen Körper }\end{array}$ & $\begin{array}{l}\text { Carter et al. } \\
(2014)[59]\end{array}$ & $\begin{array}{l}140 \text { Kinder und } \\
\text { Jugendliche }\end{array}$ & $\begin{array}{l}\text { Stichprobe aus drei ländlichen Regionen } \\
\text { mit niedrigem sozio-ökonomischem } \\
\text { Status in den USA }\end{array}$ & $\begin{array}{l}\text { T 1: } M=13,06 \\
\text { Jahre } \\
\text { T2: } M=15,37 \\
\text { Jahre }\end{array}$ & YSR; MBSRQ & Selbstbericht \\
\hline $\begin{array}{l}\text { Ausgeprägte } \\
\text { Reizbarkeit }\end{array}$ & $\begin{array}{l}\text { Burke (2012) } \\
\text { [60] }\end{array}$ & $\begin{array}{l}162 \\
\text { Jugendliche }\end{array}$ & $\begin{array}{l}\text { Klinische Stichprobe mit Jungen mit einer } \\
\text { Störung des Sozialverhaltens aus Penn- } \\
\text { sylvania und Georgia, USA }\end{array}$ & 12 - 18 Jahre & DISC & $\begin{array}{l}\text { Eltern- und } \\
\text { Lehrerbefra- } \\
\text { gung und } \\
\text { Selbstbericht }\end{array}$ \\
\hline Schlafstörungen & $\begin{array}{l}\text { Wang et al. } \\
\text { (2013) [61] }\end{array}$ & $\begin{array}{l}912 \text { Kinder und } \\
\text { Jugendliche }\end{array}$ & Stichprobe aus Shenzhen, China & $6-14$ Jahre & CSHQ, SDQ & $\begin{array}{l}\text { Elternbefra- } \\
\text { gung }\end{array}$ \\
\hline \multirow[t]{2}{*}{$\begin{array}{l}\text { Inhibition (Tempera- } \\
\text { ment mit ausgepräg- } \\
\text { ten Hemmungen) }\end{array}$} & $\begin{array}{l}\text { Mills et al. } \\
\text { (2012) [41] }\end{array}$ & 523 Kinder & $\begin{array}{l}\text { Stichprobe aus dem Concordia Longitu- } \\
\text { dinal Risk-Project und der Daycare and } \\
\text { Preschool Adjustment Study aus Kanada }\end{array}$ & 2-6 Jahre & CBQ, EAS & $\begin{array}{l}\text { Elternbefra- } \\
\text { gung }\end{array}$ \\
\hline & $\begin{array}{l}\text { Sportel et al. } \\
\text { (2013) [62] }\end{array}$ & $\begin{array}{l}1811 \\
\text { Jugendliche }\end{array}$ & $\begin{array}{l}\text { Schüler der ersten beiden Sekundar- } \\
\text { schulklassen aus den nördlichen Regio- } \\
\text { nen in den Niederlanden }\end{array}$ & $12-15$ Jahre & $\begin{array}{l}\text { RCADS; BIS; } \\
\text { ATQ }\end{array}$ & Selbstbericht \\
\hline \multirow[t]{2}{*}{$\begin{array}{l}\text { Geringe Aufmerksam- } \\
\text { keitskontrolle }\end{array}$} & $\begin{array}{l}\text { Sportel et al. } \\
\text { (2013) [62] }\end{array}$ & s. o. & & & & \\
\hline & $\begin{array}{l}\text { Brooker et al. } \\
\text { (2014) [63] }\end{array}$ & 361 Kinder & $\begin{array}{l}\text { Stichprobe mit adoptierten Kindern in } \\
\text { den USA, bei denen Elternangaben der } \\
\text { Adoptiv- und leiblichen Eltern integriert } \\
\text { wurden (Ergebnis: wenn Adoptiveltern } \\
\text { geringe eigene Angstausprägungen } \\
\text { berichten) }\end{array}$ & 9-27 Monate & $\mathrm{CBCL}$ & $\begin{array}{l}\text { Elternbefra- } \\
\text { gung }\end{array}$ \\
\hline $\begin{array}{l}\text { Einnahme von Sub- } \\
\text { stanzen (Alkohol, } \\
\text { Nikotin oder illegale } \\
\text { Drogen) }\end{array}$ & $\begin{array}{l}\text { Skrove et al. } \\
\text { (2013) [29] }\end{array}$ & $\begin{array}{l}7639 \\
\text { Jugendliche }\end{array}$ & $\begin{array}{l}\text { Population aus der, Nord Trondelag } \\
\text { Health Study` in Norwegen }\end{array}$ & 13 - 18 Jahre & SCL-5; HUNT & Selbstbericht \\
\hline $\begin{array}{l}\text { Schwere } \\
\text { Regelverstöße }\end{array}$ & $\begin{array}{l}\text { McDougall et al. } \\
\text { (2013) [64] }\end{array}$ & $\begin{array}{l}192 \\
\text { Jugendliche }\end{array}$ & $\begin{array}{l}\text { Insassen einer Jugendhaftanstalt in Nova } \\
\text { Scotia, Kanada }\end{array}$ & 13-20 Jahre & YSR & Selbstbericht \\
\hline $\begin{array}{l}\text { Intensives Grübeln (als } \\
\text { Moderator bei erhöh- } \\
\text { tem Stress) }\end{array}$ & $\begin{array}{l}\text { Michl et al. } \\
(2013)[50]\end{array}$ & $\begin{array}{l}1065 \\
\text { Jugendliche }\end{array}$ & $\begin{array}{l}\text { Schüler der Sekundarschulen (Grade } 6 \text { - } \\
\text { 8) in Central Connecticut, USA }\end{array}$ & $M=12,2$ Jahre & $\begin{array}{l}\text { CDI; MASC; } \\
\text { CRSQ; LESC }\end{array}$ & Selbstbericht \\
\hline
\end{tabular}

RCADS: Revised Child Anxiety and Depression Scale (Chorpita et al, 2005); RSES: Rosenberg Self-Esteem Scale (Rosenberg, 1965); CBQ: Children's Behavior Questionnaire (Rothbart et al., 2001); EAS: Temperament: Early developing personality traits (Buss \& Plomin, 1984); DISC: Diagnostic Interview Schedule for Children (Costello et al., 1987); BIS: Behavioral Inhibition/Behavioral Activation System Scales (Carver \& White, 1994); ATQ: Adult Temperament Questionnaire (Rothbart et al., 2000); CSHQ: Children's Sleep Habits Questionnaire (Owens et al., 2000); SDQ: Strengths and Difficulties Questionnaire (Goodman, 1997); SQC: Schema Questionnaire for Children (Stallard \& Rayner, 2005); MASC-10: Multidimensional Anxiety Scale for Children (March, 1997); CDI: Children's Depression Inventory (Kovacs, 1981); YSR: Youth-Self-Report (Achenbach, 1991); MASC: Multidimensional Anxiety Scale for Children (March, 1997); LESC: Life Events Scale for Children (Coddington, 1972); CRSQ: Children's Response Style Questionnaire (Abela et al., 2002); CDI: Children's Depression Inventory (Kovacs, 1992); SWCQ: Self-Worth Contingency Questionnaire (Burwell \& Shirk, 2006); MBSRQ: Multidimensional Body-Self Relations Questionnaire (Cash \& Grasso, 2005); CBCL: Child and Behavior Checklist (Achenbach, 1997); BASC-2: Behavioral Assessment System for Children (Reynold \& Kamphaus, 2004); OR: Odds Ratio.

${ }^{1}$ Wenn in den Studien angegeben.

2 In allen Studien werden nur die Verfahren angegeben, die für die Untersuchung der Risikofaktoren relevant sind.

${ }^{3}$ Für die Messverfahren werden im Folgenden die Namen und mindestens der Erstautor genannt; die Quellen der Messverfahren sind unter folgendem Link abrufbar: www.zkpr.unibremen.de. 
Gleichzeitig konnte das Erziehungsverhalten als wichtiger Einflussfaktor für Kinder und Jugendliche nachgewiesen werden. Bei ungünstigem Erziehungsverhalten (starke Anforderungen an die Disziplin, harsche Kritik am Kind, starke Kontrolle des Kindes) bis hin zu Nötigungen innerhalb der Familie wiesen Kinder oder Jugendliche vermehrt eine internalisierende Symptomatik auf.

Umgebungsbedingte (soziale oder umweltbezogene) Risikofaktoren sind u.a. migrationsspezifische Aspekte (wie u.a. Akkulturationsstress), die die Prävalenz für ängstliche oder depressive Symptomatik im Kindes- und Jugendalter erhöhen. Auffallend ist ebenfalls der Stellenwert der sozialen Beziehungen für Kinder und Jugendliche. Bei sozialer Ablehnung oder Mobbingerfahrungen durch Gleichaltrige entwickeln Kinder oder Jugendliche vermehrt Depression oder Angststörungen.

Unter den psychischen Risikofaktoren hat ein generelles erhöhtes Stressempfinden einen negativen Effekt auf Kinder und Jugendliche, wodurch sich die Prävalenz internalisierender Symptome bedeutsam erhöhen kann.

\section{Transdiagnostische Perspektive}

Die aufgeführten Risikofaktoren lassen sich generell mit der transdiagnostischen Perspektive vereinbaren, die Caspi et al. [23] mit ihrem p-Faktor aufgezeigt haben. Caspi und Kollegen [23] wiesen durch ihre Analysen nach, dass eine Persönlichkeit mit hohen Ängsten, negative Lebensereignisse, Zugehörigkeit zu einer niedrigen Schicht, genetische Prädispositionen und eine geringe Kontrolle der Emotionsregulation allgemein die Vulnerabilität erhöhen, unabhängig davon, welche spezifische Störung entwickelt wird. Die Studie von Caspi und Kollegen [23] hat allerdings nicht Kinder und Jugendliche untersucht. Insofern stellt sich die Frage, ob diese Perspektive auch auf das Kindes- und Jugendalter übertragbar ist. Es kann festgehalten werden, dass alle Aspekte der von Caspi und Kollegen [23] beschriebenen Risikofaktoren auch durch die systematisch recherchierten Studien dieses Reviews aufgezeigt werden können. So wird beispielsweise durch die Studien von Mills und Kollegen [41] und Sportel und Kollegen [62] deutlich, dass eine Verhaltenshemmung die Gefahr für Depression und gleichzeitig auch Angststörungen im Kindesund Jugendalter erhöhen kann. Zudem belastet Kinder und Jugendliche der Stress, der durch die Zugehörigkeit zu einer niedrigen Schicht verursacht sein kann, und führt zu einer erhöhten Prävalenz von internalisierenden Symptomen [41]. Als negative Lebensereignisse können beispielsweise plötzlich auftretende chronische oder lebensbedrohende Erkrankungen [28], körperlicher [46] oder emotionaler Missbrauch [45] von Kindern oder Jugendlichen, eine Migration [51,52] oder eine andersartige ethnische Herkunft [42] bestimmt werden, die die Gefahr für die Entwicklung von ängstlicher oder depressiver Symptomatik bei Kindern und Jugendlichen erhöhen. Außerdem kann gezeigt werden, dass selbst bei einer geringen Emotionsregulation der Mütter die Kinder vermehrt eine Depression oder Angststörungen entwickeln [41]. Kullik und Petermann [65] konnten in diesem Kontext zeigen, dass dieser Sachverhalt auch bei Jugendlichen zutrifft; Jugendliche mit Depression oder Angststörungen wenden vermehrt dysfunktionale Emotionsregulationsstrategien an.

Die vorliegenden Ergebnisse zeigen allerdings weiterführend zu Caspi und Kollegen [23], dass es im Bereich der depressiven und ängstlichen Symptomatik spezifische Risiken gibt, die ein umschriebenes Entwicklungsrisiko im Kindes- und Jugendalter darstellen. Es wird deutlich, dass mit den von Caspi et al. [23] und auch Epkins und Heckler [24] beschriebenen Faktoren nicht alle Zusammenhänge zwischen ängstlicher oder depressiver Symptomatik im Kindes- und Jugendalter erklärt werden können. So wird durch die vorliegende Studie deutlich, dass bei Kindern und Jugendlichen zusätzliche Aspekte im Vordergrund stehen und die Vulnerabilität für eine internalisierende Symptomatik erhöhen können. Unterschiedliche Studien zeigen beispielsweise, dass ein erhöhtes Stresserleben der Kinder oder ihrer Mütter zu einer erhöhten ängstlichen oder depressiven Symptomatik bei Kindern und Jugendlichen führte $[41,43,48,51,52]$. Außerdem spielt in der Kindheit und Adoleszenz die positive Qualität der sozialen Beziehungen zu Gleichaltrigen eine große Rolle (z.B. vorhandene Freundschaften, angemessene soziale Kompetenzen). Es wird beispielsweise deutlich, dass soziale Zurückweisungen bei Kindern und Jugendlichen ängstliche oder depressive Symptomatik verursachen können [33, 54, 55]. Gleichzeitig sollten Erziehungsaspekte in der Entwicklungspsychopathologie von Depression und Angststörungen nicht unterschätzt werden. Stehen übermäßige Strenge (z. B. viele Verbote) bis hin zu Nötigungen oder stark kontrollierendes Erziehungsverhalten bei Eltern im Vordergrund oder wird ein Kind vornehmlich von den Eltern kritisiert, neigen Kinder und Jugendliche beispielsweise dazu, vermehrt internalisierende Symptome zu entwickeln [41-43]. Zudem wird aber auch deutlich, dass Schlafstörungen [61] oder eine ungesunde Ernährung von Kindern oder Jugendlichen [40] ebenfalls eine stärker ausgeprägte Depression oder Angststörungen verursachen können. Die vorliegenden Ergebnisse zeigen, dass es für ängstliche oder depressive Symptomatik im Kindesund Jugendalter angemessen erscheint, die gemeinsamen Ursachen in besonderer Weise zu berücksichtigen.

\section{Bedeutung der gemeinsamen Risikofaktoren}

Es stellt sich nun die Frage, welche Bedeutung diese gemeinsamen Risikofaktoren für die Diagnostik und Therapie besitzen. Neben spezifischen Krankheitsursachen können folgende Risiken für ängstliche oder depressive Symptomatik im Kindes- und Jugendalter basierend auf den vorliegenden Ergebnissen als wesentlich herausgestellt werden:

- weibliches Geschlecht und zunehmendes Alter,

- geringe soziale Kompetenzen im Umgang mit Gleichaltrigen,

- ausgeprägtes Stresserleben,

- eine geringe Selbstwirksamkeit und

- geringe Unterstützung durch die eigene Familie.

Die transdiagnostische Perspektive geht davon aus, dass Störungen sich im Verlauf der Zeit verändern können, wenn nach wie vor bestimmte Risikofaktoren bei Kindern und Jugendlichen vorliegen. Um die Bedeutung der Risikofaktoren angemessen einordnen zu können, bedarf es weiterer Forschung. Das Vorliegen dieser gemeinsamen Risikofaktoren kann als Hinweis gewertet werden, dass es sich dabei um Ursachen mit einer stärkeren Wirkung handelt, die mit einer besonders hohen Vulnerabilität für internalisierende Probleme von Kindern und Jugendlichen einhergehen kann. Zudem besteht die Möglichkeit, dass diese gemeinsamen Risikofaktoren schneller einen ungünstigen Verlauf prognostizieren als andere Risikofaktoren. So kann vermutet werden, dass zwar beispielsweise bei häufigen Umzügen während der Kindheit die Gefahr für eine internalisierende Symptomatik steigt, aber dieser Faktor keinen gleichwertig starken Effekt aufweist wie die aufgezeigten gemeinsamen Risikofaktoren (wie beispielsweise ein geringer sozialer Rückhalt in der Familie). Gleichzeitig kann nahegelegt werden, dass im Umkehrschluss auch besonders starke Schutzfaktoren identifiziert werden müs- 
sen, um ängstlicher oder depressiver Symptomatik in der Kindheit und Adoleszenz vorzubeugen oder sie zu mindern. Möglicherweise kann das Erkennen und Behandeln von gemeinsamen Risikofaktoren dieser Störungen nachhaltiger Depression und Angststörungen im Kindes- und Jugendalter verhindern oder verringern als das sonst übliche intensive Eingehen auf vereinzelt auftretende Symptome.

\section{Implikationen für die Praxis}

Das vorliegende Review konnte viele gemeinsame Risikofaktoren für die ängstliche oder die depressive Symptomatik bei Kindern und Jugendlichen aufzeigen. Aufgrund dessen sollten nicht nur in der Ursachenforschung solche gemeinsamen Faktoren verstärkt Beachtung finden. Um diese Störungen bei Kindern und Jugendlichen angemessen zu erkennen und zu behandeln, sollte sich in der Diagnostik vermehrt auf das Auffinden dieser gemeinsamen Risikofaktoren konzentriert werden. Zudem könnten diagnostisch beide Störungen einheitlicher erfasst werden. Bei der Planung von Präventionsmaßnahmen sollten beispielsweise vermehrt ein angemessener Umgang mit Emotionen, negativen Erlebnissen und die Stärkung des Selbstbewusstseins berücksichtigt werden. Zudem können Trainings, die die Erziehungs- und Interaktionskompetenzen der Eltern verbessern, die Basis erfolgreicher Präventions- und Therapieprogramme bilden. Al-Fayez und Kollegen [66] konnten beispielsweise eine positive ElternKind-Beziehung als gemeinsamen Schutzfaktor für Kinder und Jugendliche mit Depression und Angststörungen identifizieren. Elementar erscheint in Anbetracht der Ergebnisse dieses Reviews ebenfalls die starke Relevanz der sozialen Beziehungen zu Gleichaltrigen. Da Kinder oder Jugendliche mit internalisierenden Problemen leicht übersehen werden können, sollten gemeinsam mit den Kindern und Jugendlichen beispielsweise im Gruppen-/ Schulkontext und besonders in entwicklungssensiblen Übergängen wie der Vorpubertät vermehrt emotionale und soziale Kompetenzen trainiert werden, um der Entwicklung von Depression und Angststörungen frühzeitig vorzubeugen. Dieses Review macht beispielsweise aber auch deutlich, dass bei Vorliegen von körperlichen Erkrankungen der Kinder oder Jugendlichen eine gute Compliance und ein angemessenes Coping im Umgang mit der Krankheit durch eine differenzierte Psychoedukation erfolgen müssen, um die Gefahr für Depression oder Angststörungen bei Kindern und Jugendlichen zu minimieren. Ein besonderes Augenmerk sollte ebenfalls darauf gerichtet werden, dass Kinder und Jugendliche mit psychisch kranken Eltern ein erhöhtes Risiko aufweisen, Depression oder Angststörungen zu entwickeln [38, 39, 43, 67]. Wiegand-Grefe und Kollegen [68] machen in diesem Kontext deutlich, dass besonders bei Kindern mit Eltern ohne Krankheitseinsicht und mit Persönlichkeitsstörungen die Gefahr für die Entwicklung einer psychischen Störung besonders hoch sei. Zudem implizieren die Ergebnisse dieses Reviews, dass in der psychotherapeutischen Behandlung bei Depression und Angststörungen auf vergleichbare Behandlungsmethoden zurückgegriffen werden kann [vgl. hierzu 69].

\section{Take Home Message}

Depressive und ängstliche Symptomatik bei Kindern und Jugendlichen wird beeinflusst von gemeinsamen Risikofaktoren. Eine systematische Literaturrecherche kann in diesem Kontext 44 Risikofaktoren identifizieren, u.a. das weibliche Geschlecht, das Einsetzen der Pubertät, den Umgang mit chronischen Erkrankungen, erhöhtes Stresserleben von Kindern oder Müttern, negatives Erziehungsverhalten der Eltern. Eine ähnliche Behandlung der depressiven und ängstlichen Symptomatik wird nahegelegt.

Interessenkonflikt: Die Autoren geben an, dass kein Interessenkonflikt besteht.

\section{Literatur}

1 Costello EJ, Egger HL, Copeland W et al. The developmental epidemiology of anxiety disorders: phenomenology, prevalence, and comorbidity. In: Silverman W, Field AP ed. Anxiety disorders in children and adolescents. 2nd ed. Cambridge: Cambridge University Press; 2011: 56 - 75

2 Groen G, Petermann F. Was wirkt in der Therapie depressiver Kinderund Jugendlicher wirklich? Kindh Entwickl 2008; 17: 242 -250

3 Merinkangas KR, He JP, Burstein M et al. Lifetime Prevalence of Mental Disorders in U.S. Adolescents: Results from the National Comorbidity Survey Replication-Adolescent Supplement (NCS-A). J Am Acad Child Adolesc Psychiatry 2010; 49: 980 -989

4 Dobson KS, Dozois DA. Risk factors in depression. San Diego: Elsevier; 2008

5 Essex MJ, Klein MH, Slattery MJ et al. Early risk factors and development pathways to chronic inhibition and social anxiety disorder in adolescence. Am J Psychiatry 2010; 167: 40-46

6 Laskey B, Cartwright-Hatton S. (2012) Childhood anxiety and depression. In: Davey G ed. Clinical Psychology. New York: Routledge; 2012: $71-86$

7 American Psychiatric Association. Diagnostic and Statistical Manual of Mental disorders. (5th ed.). Arlington (VA): American Psychiatric Association; 2013

8 Costello EH, Egger HL, Angold A. Developmental epidemiology of anxiety disorders. In: Ollendick TH ed. Phobic and anxiety disorders in children and adolescents: A Clinicians guide to effective psychosocial and pharmacological interventions. New York: Oxford University Press; 2004: 61-91

9 Keren M, Tyano S. Depression in Infancy. Child and Adolescent Psychiatry Clinic in North American Countries 2006; 15: 883-897

10 Hölling H, Schlack R, Petermann F et al. Psychische Auffälligkeiten und psychosoziale Beeinträchtigungen bei Kindern und Jugendlichen im Alter von 3 bis 17 Jahren in Deutschland. Prävalenz und zeitliche Trends zu 2 Erhebungszeitpunkten (2003-2006 und 2009-2012). Bundesgesundheitsblatt Gesundheitsforschung Gesundheitsschutz 2014; 57: $807-819$

11 Beesdo K, Knappe S, Pine DS. Anxiety and anxiety disorders in children and adolescents: developmental issues and implications for DSM-V. Psychiatr Clin North Am 2009; 32: 483-524

12 Faedda GL, Serra G, Marangoni C et al. Clinical risk factors for bipolar disorders: a systematic review of prospective studies. J Affect Disorders 2014; 168: 314-321

13 Lammers F, van Oppen P, Comijcs C et al. Comorbidity patterns of anxiety and depressive disorders in a large cohort study: the Netherlands study of depression and anxiety (NESDA). J Clin Psychiatry 2011; 72: $341-348$

14 Matthew AR, Pettit JW, Lewinsohn PM et al. Co-morbidity between major depression and anxiety disorders: shared etiology or direct causation? Psychol Med 2011; 41: 2023 - 2034

15 Ehrenreich-May J, Chu BC. Overview of transdiagnostic mechanisms and treatments for youth psychopathology. In: ed. Ehrenreich-May J, Chu BC. Transdiagnostic treatments for children and adolescents. New York: Guilford Press; 2014: 3-14

16 Chu BC. Translating transdiagnostic approaches to children and adolescents. Cogn Behav Pract 2012; 19: 1 -4 
17 Barlow DH, Allen LB, Choate ML. Toward a unified treatment for emotional disorders. Behav Ther 2004; 35: 205-230

18 Barlow DH, Farchione TF, Fairholme CP et al. Unified protocol for the transdiagnostic treatment of emotional disorders: Therapists guide. New York: Oxford University Press; 2010

19 Southam-Gerow MA, Kendall PC. Emotion regulation and understanding implications for child psychopathology and therapy. Clin Psychol Rev 2002; 22: 189-222

20 Compas BE, Watson KH, Reising MM et al. Stress and coping in child and adolescent psychopathology. In: Ehrenreich-May J, Chu BC ed. Transdiagnostic treatments for children and adolescents. New York: Guilford Press; 2014: $35-58$

21 Nolen-Hoeksema S, Watkins ER. A heuristic for developing transdiagnostic models of psychopathology: Explaining multifinality and divergent trajectories. Perspect Psychol Sci 2011; 6: 589-609

22 Dozois DJA, Seeds PM, Collins KA. (2009) Transdiagnostic approaches to the prevention of depression and anxiety. J Cognit Psychother 2009; 23: $44-59$

23 Caspi A, Houts RM, Belsk DW et al. The p Factor: One general psychopathology factor in the structure of psychiatric disorders? Clin PsycholSci Pra 2014; DOI: 10.1177/2167702613497473

24 Epkins CC, Heckler D. Integrations etiological models of social anxiety and depression in youth: evidence for a cumulative interpersonal model. Clin Child Fam Psychol Rev 2011; 14: 329- 376

25 Centre for Reviews and Dissemination. Systematic Reviews: CRD's guidance for undertaking reviews in health care. York: University of York; 2009

26 ICD-10: International Statistical Classification of Diseases and Health Related Problems. Tenth Revision (ICD-10), Volume III Alphabetical Index. World Health Organisation; 1994

27 American Psychiatric Association. Diagnostic and Statistical Manual of Mental disorders. (4th ed.). Arlington (VA): American Psychiatric Association; 2000

28 O'Dell SM, DuPaul GJ. Predictors of emotional problems in children with diabetes mellitus. Child Health Care 2012; 41: 32 - 42

29 Skrove M, Romundstad P, Indrevadik MS. Resilience, lifestyle and symptoms of anxiety and depression in adolescence: the YOUNG-HUNT study. Soc Psych Psych Epid 2013; 48: 407-416

30 De Jong PJ, Sportel BE, de Hullu E et al. Co-occurrence of social anxiety and depression symptoms in adolescence: differential links with implicit and explicit self-esteem? Psychol Med 2012; 42: 473-481

31 Slopen N, Fitzmaurice GM, Williams DR et al. Common patterns of violence experiences and depression and anxiety among adolescents. Soc Psych Psych Epid 2012; 47: 1591 - 1605

32 Bean KF. Differential ratings of and maternal impact on anxiety and depression among African American children in special education. Child Youth Serv Rev 2012; 34: $1868-1875$

33 Ghoul A, Niwa EY, Boxer P. The role of contingent self-worth in the relation between victimization and internalizing problems in adolescents. J Adolescence 2013; 36: 457-464

34 Glasheen C, Richardson GA, Kim KH et al. Exposure to maternal pre- and postnatal depression and anxiety symptoms: Risk for Major depression, anxiety disorders, and conduct disorder in adolescent offspring. Dev Psychopathol 2013; 25: 1045-1063

35 Lavigne JV, Herzing LBK, Cook EH et al. Gene × Environment effects of serotonin transporter, dopamine receptor $\mathrm{D} 4$, and monoamine oxidase A genes with contextual and parenting risk factors on symptoms of oppositional defiant disorder, anxiety, and depression in a community sample of 4-year-old children. Dev Psychopathol 2013; 25: 555-575

36 Herringa RJ, Birn RM, Ruttle PL et al. Childhood maltreatment is associated with altered fear circuity and increased internalizing symptoms by late adolescence. Neuroscience 2013; 110: 19119-19124

37 Imran N, Ani C, Mahmood Z et al. Anxiety and depression predicted by medically unexplained symptoms in Pakistani children: A case-control study. J Psychosom Res 2014; 76: 105-112

38 Mendes AV, de Souza Crippa JA, Souza RM et al. Risk factors for mental health problems in school-aged children from a community sample. Matern Child Health 2013; 17: 1825-1834

39 Nadia M, De Stavola B, Ploubidis GB et al. The effects of maternal eating disorders on offspring childhood and early adolescent psychiatric disorders. Int J Eat Disorder 2014; 47: 385-393

40 Jacka FN, Ystrom E, Brantsaeter AL et al. Maternal and early postnatal nutrition and mental health of offspring by age 5 years: a prospective cohort study. J Am Acad Child Adolesc Psychiatry 2013; 52: 1038 1047
41 Mills RSL, Hastings PD, Helm J et al. Temperamental, parental, and contextual contributors to early-emerging internailizing problems: a new integrative analyses approach. Soc Dev 2011; 21: 229-253

42 Huang KY, Cheng S, Calzada E et al. Symptoms of anxiety and associated risk and protective factors in young Asian American children. Child Psychiat Hum Dev 2012; 43: 761 - 774

43 Bayer JK, Ukoumunne OC, Mathers $M$ et al. Development of children's internalising and externalising problems from infancy to five years of age. Australian \& New Zealand J Psychiat 2012; 46: 659-668

44 Glasheen C, Richardson GA, Kim KH et al. Exposure to maternal pre- and postnatal depression and anxiety symptoms: Risk for major depression, anxiety disorders, and conduct disorder in adolescent offspring. Dev Psychopathol 2013; 25: 1045 - 1063

45 Hamilton JL, Shapero BG, Stange JP et al. Emotional maltreatment, peer victimization, and depressive versus anxiety symptoms during adolescence: hopelessness as a mediator. J Clin Child Psychol 2013; 42: 332 347

46 Pajer KA, Gardner W, Lourie A et al. Physical child abuse potential in adolescent girls: associations with psychopathology, maltreatment, and attitudes toward child-bearing. Psychiatry 2014; 59: 98-106

47 Espejo PE, Hammen C. Elevated appraisals of the negative impact of naturally occuring life events: a risk factor of depressive and anxiety disorders. J Abnorm Child Psychol 2012; 40: 303-315

48 Barber BA, Kohl KL, Kassam-Adams N et al. Acute stress, depression, and anxiety symptoms among english and spanish speaking children with recent trauma exposure. J Clin Psychol Med S 2014; 21: 66 - 71

49 Uliaszek AA, Zinbarg RE, Mineka $S$ et al. A longitudinal examination of stress generation in depressive and anxiety disorders. J Abnorm Psychol 2012; 121: 4-15

50 Michl LC, McLaughlin KA, Sheperd K et al. Rumination as a mechanism linking stressful life events to symptoms of depression and anxiety: longitudinal evidence in early adolescents and adults. J Abnorm Psychol 2013; 122: 339-352

51 Sirin SR, Gupta T, Ryce P et al. Understanding the role of social support in trajectories of mental health symptoms for immigrant adolescents. J Appl Dev Psychol 2013; 34: 199-207

52 Vervilet M, Meyer Dermott MA, Jakobsen $M$ et al. The mental health of unaccompanied refugee minors on arrival in the host country. Health Disabil 2014; 55: 33-37

53 Slopen N, Fitzmaurice GM, Williams DR et al. Common patterns of violence experiences and depression and anxiety among adolescents. Soc Psych Psych Epid 2012; 47: 1591 - 1605

54 Lauer BAM, Renk $K$. The peer informant: characteristics related to the perceptions of peer behavior problems. J Child Fam Stud 2013; 22: $786-800$

55 Fite PJ, Rubens SL, Preddy TM et al. Reactive/proactive aggression and the development of internalizing problems in males: the moderating effect of parent and peer relationships. Aggressive Behav 2014; 40: $69-78$

56 Myers RM, Balsamo L, Lu X et al. A prospective study of anxiety, depression, and behavioral changes in the first year after a diagnosis of childhood acute lymphoblastic leukemia. Cancer 2014; 120: 1417-1425

57 Van Tuijl LA, de Jong PJ, Sportel BE et al. Implicit and explicit self-esteem and their reciprocal relationship with symptoms of depression and social anxiety: A longitudinal study in adolescents. J Behav Ther Exp Psy 2014; 45: $113-121$

58 Keyfitz L, Lumley MN, Hennig KH et al. The role of positive schemas in child psychopathology and resilience. Cognitive Ther Res 2013; 37: $97-108$

59 Smith CarterJ, Smith S, Bostick S et al. Mediating effects of parent-child relationships and body image in the prediction of internalizing symptoms in urban youth. J Youth Adolesc 2014; 43: 554-567

60 Burke JD. An affective dimension within ODD symptoms among boys: personality and psychopathology outcomes into early adulthood. J Child Psychol Psychiatry 2012; 53: 1176-1183

61 Wang GH, Xu GX, Liu Z et al. Sleep patterns and sleep disturbances among Chinese school-aged children: prevalence and associated factors. Sleep Med 2013; 14: 45-52

62 Sportel BE, Nauta MH, de Hullu E et al. Predicting internailizing symptoms over a two year period by BIS, FFFS and attentional control. Pers Indiv Differ 2013; 54: 236-240

63 Brooker RJ, Neiderhiser JM, Ganiban JM et al. Birth and adoptive parent anxiety symptoms moderate the link between infant attention control and internalizing problems in toddlerhood. Dev Psychopathol 2014; 26: $347-359$ 
64 McDougall A, Campbell MA, Santor DA. Institutional offense patterns in adolescent offenders: the role of antisocial and mental health indicators. Youth Viol Juven Just 2013; 00: 1 - 16

65 Kullik A, Petermann F. Dysfunktionale Emotionsregulation als grundlegendes Merkmal von Jugendlichen mit Angst- und depressiven Störungen. Fortschr Neurol Psychiat 2013; 81: 35 - 39

66 Al-Fayez GA, Ohaeri JU, Gado OM. Prevalence of physical, psychological, and sexual abuse among a nationwide sample of Arab high school students: association with family characteristics, anxiety, depression, self-esteem, and quality of life. Soc Psych Psych Epid 2012; 47: 53-66
67 Wiegand-Grefe S, Cronemeyer B, Plass A et al. Psychische Auffälligkeiten von Kindern psychisch kranker Eltern: ein Perspektivenvergleich. Kindh Entwickl 2013; 22: $31-40$

68 Wiegand-Grefe S, Geers P, Petermann F et al. Kinder psychisch kranker Eltern: Merkmale elterlicher psychischer Erkrankung und Gesundheit der Kinder aus Elternsicht. Fortschr Neurol Psychiat 2011; 79: 32-40

69 Hoffmann F, Glaeske G, Petermann F et al. Outpatient treatment in German adolescents with depression: an analysis of nationwide health insurance data. Pharmacoepidem Dr S 2012; 21: 972 - 979 